University of Nebraska - Lincoln

DigitalCommons@University of Nebraska - Lincoln

Biological Systems Engineering: Papers and

Publications

Biological Systems Engineering

8-1978

\title{
Micro-Relief Surface Depression Storage: Changes During Rainfall Events And Their Application To Rainfall-Runoff Models
}

J. Kent Mitchell

University of Illinois, Urbana-Champaign, jkmitche@illinois.edu

Benjamin A. Jones Jr.

University of Illinois, Urbana-Champaign

Follow this and additional works at: https://digitalcommons.unl.edu/biosysengfacpub

Part of the Biological Engineering Commons

Mitchell, J. Kent and Jones, Benjamin A. Jr., "Micro-Relief Surface Depression Storage: Changes During Rainfall Events And Their Application To Rainfall-Runoff Models" (1978). Biological Systems Engineering: Papers and Publications. 291.

https://digitalcommons.unl.edu/biosysengfacpub/291

This Article is brought to you for free and open access by the Biological Systems Engineering at DigitalCommons@University of Nebraska - Lincoln. It has been accepted for inclusion in Biological Systems Engineering: Papers and Publications by an authorized administrator of DigitalCommons@University of Nebraska Lincoln. 
Reprinted from Volume 14 - Number 4 - August 1978
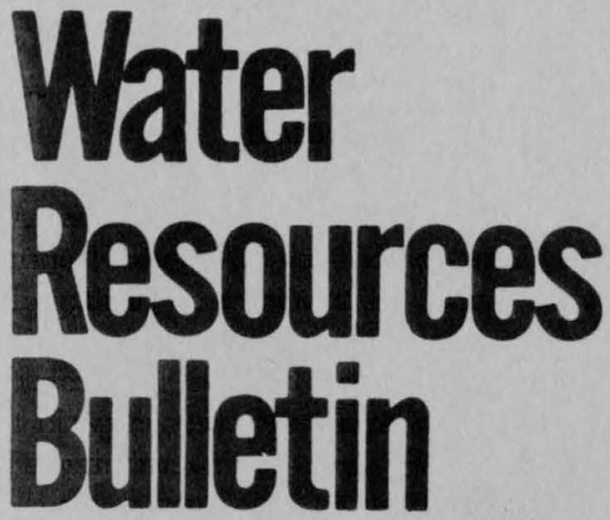

American Water Resources Association 


\title{
MICRO-RELIEF SURFACE DEPRESSION STORAGE: CHANGES DURING RAINFALL EVENTS AND THEIR APPLICATION TO RAINFALL-RUNOFF MODELS ${ }^{1}$
}

\author{
J. Kent Mitchell and Benjamin A. Jones, Jr. ${ }^{2}$
}

\begin{abstract}
Micro-relief surface depression storage is one of the dynamic components of the rainfall-runoff process. The quantification of the effect of rainfall intensity and duration on the micro-relief was the subject of this study. Micro-relief measurements were made on 88 soil bin samples before and after the application of simulated rainfall events. The surface depression changes are described with empirical equations, using basic rainfall, surface hydrology, and soil parameters and their cross products as independent variables. A rainfall-runoff model demonstrates the value of a dynamic description of the surface depression storage function.

(KEY TERMS : hydrology; micro-relief; surface storage; depression storage; rainfall simulation; hydrologic modeling.)
\end{abstract}

In the rainfall-runoff process, water, in the form of rainfall, is delivered to the surface of the earth in amounts that vary spatially and temporally. A part of this rainfall is intercepted and held by the vegetal cover for future disposition through evaporation. The water that reaches the ground infiltrates into the soil at a rate dependent upon the infiltration capacity of the soil at that time. If the rainfall intensity exceeds the infiltration capacity of the soil, the micro-relief surface depressions will be filled. When this depression storage is filled and exceeded, the water level builds up on the surface and runoff begins. The surface runoff continues to watershed elements of lower elevation depending upon the rainfall, infiltration, and micro- and macro-relief of successively lower elements.

All of the phenomena may proceed at varying rates depending upon the physical conditions. For instance, if rainfall decreases to a sufficiently low rate, infiltration may exceed rainfall and depression storage will be drained. Then, when the next burst of rainfall occurs, the depression storage must be replenished before surface runoff can begin. Conversely, rainfall may continue at a rate large enough to keep the surface depressions full until the storm ends. In that case the depression storage is filled only once during a storm rather than several times.

${ }^{1}$ Paper No. 77086 of the Water Resources Bulletin. Discussions are open until April 1, 1979. This study was a part of Project 10-315 of the Agricultural Experiment Station, College of Agriculture, University of Illinois at Urbana-Champaign.

${ }^{2}$ Respectively, Assistant Professor and Professor, Agricultural Engineering Department, University of Illinois, Urbana-Champaign, Illinois. 
A number of investigators have developed models that evaluate many hydrologic phenomena separately within discrete subareas to obtain a prediction of the runoff hydrograph for a watershed. Such models were presented by Crawford and Linsley (1966), Huggins and Monke (1966), Holtan, et al. (1975), and Massie (1975). The effect of the various phenomena are evaluated for each subarea as are their effect on adjacent subareas. The resulting runoff is accumulated area by area in small time increments to obtain a runoff hydrograph at the outlet. Many of the conceptual models contain sections describing the effect of surface depression storage, but few present quantitative data adequate to describe values of surface depression storage either before or during a rainfall event.

This paper describes studies conducted to determine the effect of rainfall intensity and duration on micro-relief surface depression storage. Prediction equations are developed to describe the change in the maximum micro-relief surface depression depth, the microrelief surface depression storage, and the depth-storage model parameters as affected by rainfall, surface hydrology, and soil variables. Methods of application of the equations are suggested and a basic rainfall-runoff model demonstrates the effects of a dynamic description of the surface depression storage function.

\section{EQUIPMENT}

To study the effect of rainfall amount and duration on the change in micro-relief surface depression storage, it was necessary to control the rainfall production, the soil surface before rainfall, and the measurement of the soil surface before and after rainfall. The equipment was located in a 32 -foot drop tower as described by Bubenzer (1970), Bubenzer and Jones (1971), and Mitchell (1970). The rainfall production system consisted of drop modules that produced drops with a mean diameter of $3.42 \mathrm{~mm}$ and provided rainfall rates of 3 to 15 inches per hour. A 3 -foot square soil bin with soil depth of twelve inches, and runoff and percolation tanks and recorders to give time-depth relationships were located at the bottom of the tower.

The soil surfaces were measured using a profile measuring device (PMD) that was developed at the University of Illinois specifically for this study. Details of construction and operation and the preliminary testing and calibration were described by Mitchell (1970) and Mitchell and Jones (1973). Basically the PMD automatically measured, on a 1 -inch grid, the height of points in a 36 -inch by 36 -inch square.

\section{PRELIMINARY STUDIES}

Four modules were used to produce the simulated rainfall. An analysis of the variability of flow rate between the modules showed that the rainfall rate was equal.

The simulated rainfall was analyzed to determine the drop diameters produred and the variation in amounts and intensities as described by Mitchell (1970). At the mean intensity of 9.48 inches per hour the mean drop diameter was $3.42 \mathrm{~mm}$ and the drop diameter increased (decreased) $0.056 \mathrm{~mm}$ for each unit of rainfall intensity increase (decrease)from the mean.

Tests were made using material from four major soils of Illinois: Flanagan and Cisne silt loams, Sparta loamy sand, and Darwin silty clay. 
Flanagan silt loam is a member of the Sidell, Catlin, Flanagan, Drummer association of soils which were formed under grass vegetation. This soil is a black prairie soil formed from thirty-six to sixty inches of loess over Wisconsin till. It occurs on slopes of 1 to 3 percent.

Cisne silt loam is a brownish-gray upland soil developed under grass vegetation on slopes of less than $1 \frac{1 / 2}{2}$ percent. This soil occurs in association with Huey silt loam and Hoyleton silt loam. It was formed from loess less than 45 inches thick over weathered Illinoian till.

Sparta loamy sand is a moderately dark colored terrace soil developed under grass vegetation on slopes ranging from 1 to 7 percent.

Darwin silty clay is one of the soils found in stream valleys in Illinois. The surface is a very dark gray silty clay overlaying a gray to olive-gray silty clay subsoil. Slopes range from 0 to 2 percent.

Extensive soil samples were taken at each site for laboratory analysis. Mechanical analyses of the soil were made to determine the proportion of sand, silt, and clay. The results are shown in Table 1.

TABLE 1. Particle Size Distribution.

\begin{tabular}{|c|c|c|c|}
\hline \multirow[b]{2}{*}{ Soil } & \multicolumn{3}{|c|}{ Particle Size Distribution } \\
\hline & Sand $(>0.05 \mathrm{~mm})$ & Silt & Clay $(<0.002 \mathrm{~mm})$ \\
\hline Flanagan silt loam & 10 & 61 & 29 \\
\hline Cisne silt loam & 20 & 61 & 19 \\
\hline Sparta loamy sand & 81 & 11 & 8 \\
\hline Darwin silty clay & 20 & 36 & 44 \\
\hline
\end{tabular}

\section{METHODS}

\section{Experimental Design}

A bin of soil was subjected to rainfall intensities of $3,6,9,12$, or 15 inches per hour and durations of $10,20,30$, or 50 minutes for most intensity-duration combinations. Two soil surfaces were tested, one medium and one rough. The order of runs within a soil type was selected randomly.

Eighty-eight runs were conducted: 20 on Flanagan silt loam, 24 on Cisne silt loam, 20 on Sparta loamy sand, and 24 on Darwin silty clay. The 15 iph intensity was abandoned after observing the results on Flanagan silt loam. Runs were not conducted on the Sparta soil at 12 iph intensity for greater than 10 minutes duration or for 9 iph intensity50 minute duration because the Sparta soil became nearly flat at medium intensityduration combinations.

\section{Test Procedure}

A complete test run consisted of many different steps and measurements which are summarized here. The detailed procedure is presented by Mitchell (1970). 
The simulated rainfall was started at the desired intensity, and the system was allowed a minimum of 30 minutes to reach equilibrium conditions. Rainfall was collected and measured in a 3-x 3-foot raingage for a period of approximately one hour to determine the exact rainfall intensity produced.

The soil being tested was tilled with hand tools to a depth of approximately 8 inches to prepare the desired roughness. After tillage, a soil moisture sample was taken, the soil bin was weighed, and the initial soil surface configuration was measured with the PMD. Next the soil bin with its splash shield was covered and placed in the drop tower, and the runoff and percolation collection tanks were connected. Finally, the soil bin cover was removed and the duration timing begun. At the end of the specified rainfall duration the cover was replaced and the covered soil bin was left in the drop tower until runoff ceased and percolation decreased to a small rate. When the soil bin was removed from the drop tower, another rainfall intensity measurement and soil moisture sample were taken and the soil bin was weighed. Then the final soil configuration was measured using the PMD.

\section{Data Reduction}

Six samples were taken from each simulated rainfall test to determine rainfall intensity. Water level recorder charts were transcribed to obtain peak and volume of both runoff and percolation.

The PMD data were processed to obtain depth-storage values on the assumption that each point measurement was the center of a 1-inch square level surface as described by Mitchell and Jones (1976):

$$
\mathrm{S}_{\mathrm{r}}=\sum_{\mathrm{i}=1}^{\mathrm{m}} \sum_{\mathrm{j}=1}^{\mathrm{n}}\left(\mathrm{H}_{\mathrm{r}}-\mathrm{H}_{\mathrm{a}}\right)
$$

where:

$\mathrm{S}_{\mathrm{r}} \quad=$ surface storage below a reference height, cubic inches;

$\mathrm{i}, \mathrm{j}=$ rows and columns of point measurements, respectively;

$\mathrm{H}_{\mathrm{r}}=$ reference height, inches, and

$\mathrm{H}_{\mathrm{a}}=$ point measurement of height on the soil surface, inches.

The average surface storage, in inches, at any reference height is, then, the accumulative storage computed by Equation (1), divided by the total surface area.

The depth-storage data from each PMD run were then fitted with the depth-storage model selected by Mitchell and Jones (1976):

$$
\mathrm{S}=\mathrm{aD}^{\mathrm{b}}
$$

where:

$\mathrm{S} \quad=$ storage, inches;

$\mathrm{D}=$ depth above the lowest point on the surface, inches; and 


\section{$a, b=$ equation parameters.}

The soil bin weight before and after a test, the rainfall applied, and the runoff and percolation volumes were used to indicate the accuracy of the overall water measurement system. This relationship is described as follows:

$$
\mathrm{w}_{\mathrm{b}}+\mathrm{R}_{\mathrm{a}}-\mathrm{R}_{\mathrm{u}}-\mathrm{P}-\mathrm{w}_{\mathrm{a}}=\mathrm{e}
$$

where:

$$
\begin{aligned}
& \mathrm{W}_{\mathrm{b}}=\text { weight of soil bin before rainfall, } \\
& \mathrm{R}_{\mathrm{a}} \quad=\text { weight of rainfall applied, } \\
& \mathrm{R}_{\mathrm{u}} \quad=\text { weight of runoff collected, } \\
& \mathrm{P} \quad=\text { weight of percolation collected, } \\
& \mathrm{W}_{\mathrm{a}} \quad=\text { weight of soil bin after rainfall, and } \\
& \mathrm{e} \quad=\text { discrepancy. }
\end{aligned}
$$

The discrepancy was converted to inches of water over the soil surface and was further described as a percentage of the rainfall applied. The discrepancy range was 0.03 to 36.4 percent, but the average discrepancy was 6.1 percent with only 10 of the 88 runs greater than 10 percent.

Infiltration volumes were obtained indirectly, using the rainfall applied, the runoff volume, and the depression storage at the end of the rainfall period. If runoff was occurring at the end of the rainfall period, the infiltration volume was calculated as the rainfall applied minus the runoff volume minus the volume of depression storage to the depth of equal cut-and-fill. If runoff did not occur, the infiltration volume was considered to be equal to the rainfall volume.

\section{RESULTS AND DISCUSSION}

The controlled rainfall intensity and duration used in the study were considered first in the analyses. An initial study using Flanagan silt loam (Mitchell, 1970) showed that these two variables explained 40 to 50 percent of the variation in the soil surface configuration. Rainfall energy, momentum and mass relationships were also considered in the initial investigation of significant independent variables. However, they did not improve the description of soil surface configuration changes when compared to rainfall intensity and duration as independent variables.

Visual representation of a soil surface is shown in Figure 1. The computer drawn figure shows the soil surface before and after the approximately 9-iph intensity, 30 minute rainfall for one repetition of the Cisne silt loam soil test.

The extent of the changes in maximum depth and maximum storage with rainfall intensity and duration changes for each soil type tested are presented in Tables 2 through 5 . Most intensity-duration combinations had two repetitions - one for a medium and one 


\section{CISNE SILT LOAM}
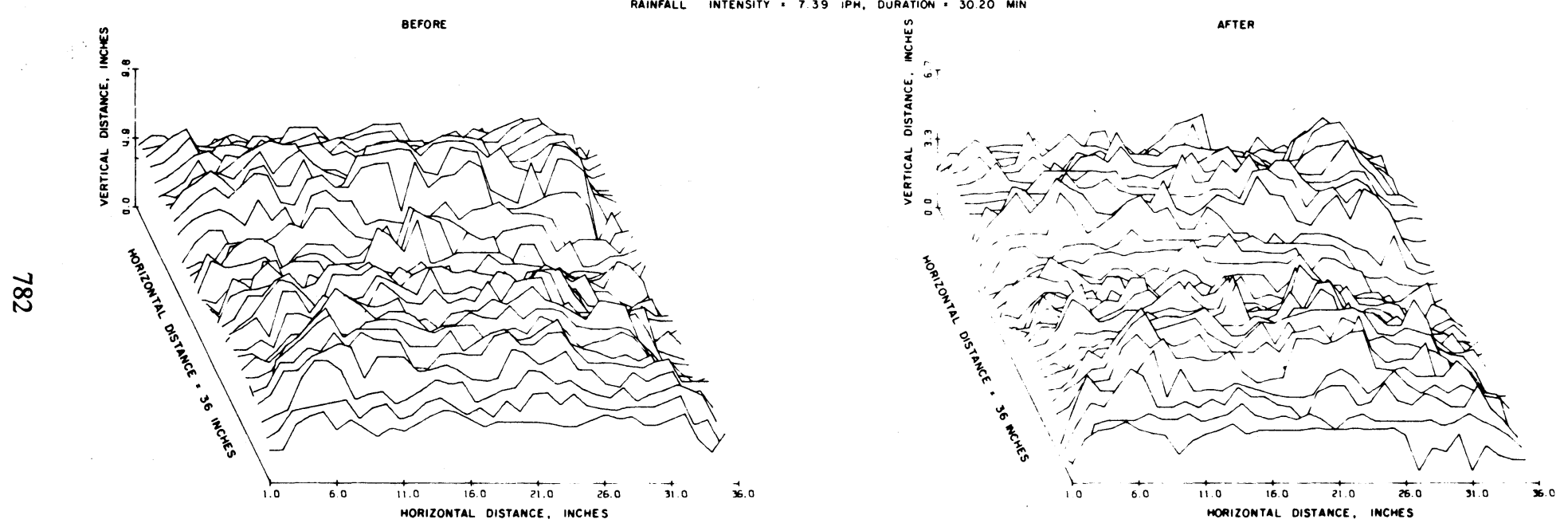

Figure 1. Computer-Drawn Representation of a Cisne Silt Loam Soil Test. 
TABLE 2. Micro-Relief Maximum Depth and Storage Before and After Rainfall: Ilanagan Silt Loam.

\begin{tabular}{|c|c|c|c|c|c|c|c|c|c|c|c|c|c|c|}
\hline \multirow{3}{*}{$\begin{array}{c}\text { Rainfall } \\
\text { Duration } \\
\text { (Min.) }\end{array}$} & \multirow[b]{3}{*}{$\begin{array}{c}\text { Surface } \\
\text { Condition }\end{array}$} & \multirow{3}{*}{$\begin{array}{l}\text { Maximum } \\
\text { Depth, } D_{m} \text {, } \\
\text { or Max. } \\
\text { Storage, } S_{m}\end{array}$} & \multicolumn{12}{|c|}{ Approximate Rainfall Intensity, iph } \\
\hline & & & \multicolumn{3}{|c|}{3} & \multicolumn{3}{|c|}{6} & \multicolumn{3}{|c|}{9} & \multicolumn{3}{|c|}{15} \\
\hline & & & $\begin{array}{c}\text { Before } \\
\text { (inches) }\end{array}$ & $\begin{array}{c}\text { After } \\
\text { (inches) }\end{array}$ & $\begin{array}{c}\text { Decrease } \\
\text { (inches) }\end{array}$ & $\begin{array}{c}\text { Before } \\
\text { (inches) }\end{array}$ & $\begin{array}{c}\text { After } \\
\text { (inches) }\end{array}$ & $\begin{array}{c}\text { Decrease } \\
\text { (inches) }\end{array}$ & $\begin{array}{l}\text { Before } \\
\text { (inches) }\end{array}$ & $\begin{array}{c}\text { After } \\
\text { (inches) }\end{array}$ & $\begin{array}{c}\text { Decrease } \\
\text { (inches) }\end{array}$ & $\begin{array}{l}\text { Before } \\
\text { (inches) }\end{array}$ & $\begin{array}{c}\text { After } \\
\text { (inches) }\end{array}$ & $\begin{array}{r}\text { Decrease } \\
\text { (inches) }\end{array}$ \\
\hline \multirow[t]{4}{*}{10} & Rough & $\mathrm{D}_{\mathrm{m}}$ & 9.3 & 8.4 & 0.9 & & & & 8.0 & 6.6 & 1.4 & 7.4 & 6.4 & 1.0 \\
\hline & & $\mathrm{S}_{\mathrm{m}}$ & 5.21 & 4.74 & 0.47 & & & & 3.14 & 2.68 & 0.46 & 4.17 & 3.22 & 0.95 \\
\hline & Medium & $\mathrm{D}_{\mathrm{m}}$ & 7.0 & 5.8 & 1.2 & 6.6 & 6.1 & 0.5 & 6.2 & 4.5 & 1.7 & 6.6 & 3.8 & 2.8 \\
\hline & & $\mathrm{S}_{\mathrm{m}}$ & 3.87 & 2.94 & 0.93 & 3.62 & 3.27 & 0.35 & 3.44 & 2.03 & 1.41 & 3.42 & 1.43 & 1.99 \\
\hline \multirow[t]{4}{*}{30} & Rough & $\mathrm{D}_{\mathrm{m}}$ & 8.4 & 7.5 & 0.9 & & & & 9.0 & 6.8 & 2.2 & 10.5 & 8.5 & 2.0 \\
\hline & & $\mathrm{S}_{\mathrm{m}}$ & 4.74 & 3.92 & 0.82 & & & & 4.96 & 3.52 & 1.44 & 5.04 & 3.85 & 1.19 \\
\hline & Medium & $\mathrm{D}_{\mathrm{m}}$ & 5.8 & 5.4 & 0.4 & 6.6 & 4.7 & 1.9 & 6.3 & 5.5 & 0.8 & & & \\
\hline & & $\mathrm{S}_{\mathrm{m}}$ & 2.88 & 2.61 & 0.27 & 2.65 & 1.92 & 0.73 & 3.24 & 2.55 & 0.69 & & & \\
\hline \multirow[t]{6}{*}{50} & Rough & $\mathrm{D}_{\mathrm{m}}$ & 8.6 & 6.0 & 2.6 & & & & 8.0 & 5.3 & 2.7 & & & \\
\hline & & $\mathrm{S}_{\mathrm{m}}$ & 4.45 & 2.54 & 1.91 & & & & 3.48 & 1.99 & 1.49 & & & \\
\hline & Medium & $\mathrm{D}_{\mathrm{m}}$ & 5.7 & 4.0 & 1.7 & 5.8 & 4.3 & 1.5 & 6.9 & 4.5 & 2.4 & 6.6 & 3.7 & 2.9 \\
\hline & & $\mathrm{S}_{\mathrm{m}}$ & 2.78 & 1.79 & 0.99 & 2.62 & 1.97 & 0.65 & 4.15 & 2.38 & 1.77 & 3.05 & 0.69 & 2.36 \\
\hline & Medium & $\mathrm{D}_{\mathrm{m}}$ & & & & & & & 6.5 & 4.1 & 2.4 & & & \\
\hline & & $\mathrm{S}_{\mathrm{m}}$ & & & & & & & 3.85 & 2.07 & 1.78 & & & \\
\hline
\end{tabular}


TABLE 3. Micro-Relief Maximum Depth and Storage Before and After Rainfall: Cisne Silt Loam.

\begin{tabular}{|c|c|c|c|c|c|c|c|c|c|c|c|c|c|c|}
\hline \multirow{3}{*}{$\begin{array}{c}\text { Rainfall } \\
\text { Duration } \\
\text { (Min.) }\end{array}$} & \multirow[b]{3}{*}{$\begin{array}{c}\text { Surface } \\
\text { Condition }\end{array}$} & \multirow{3}{*}{$\begin{array}{l}\text { Maximum } \\
\text { Depth, } \mathrm{D}_{\mathrm{m}} \text {, } \\
\text { or Max. } \\
\text { Storage, } \mathrm{S}_{\mathrm{m}}\end{array}$} & \multicolumn{12}{|c|}{ Approximate Rainfall Intensity, iph } \\
\hline & & & \multicolumn{3}{|c|}{3} & \multicolumn{3}{|c|}{6} & \multicolumn{3}{|c|}{9} & \multicolumn{3}{|c|}{12} \\
\hline & & & $\begin{array}{c}\text { Before } \\
\text { (inches) }\end{array}$ & $\begin{array}{c}\text { After } \\
\text { (inches) }\end{array}$ & $\begin{array}{l}\text { Decrease } \\
\text { (inches) }\end{array}$ & $\begin{array}{c}\text { Before } \\
\text { (inches) }\end{array}$ & $\begin{array}{c}\text { After } \\
\text { (inches) }\end{array}$ & $\begin{array}{l}\text { Decrease } \\
\text { (inches) }\end{array}$ & $\begin{array}{c}\text { Before } \\
\text { (inches) }\end{array}$ & $\begin{array}{c}\text { After } \\
\text { (inches) }\end{array}$ & $\begin{array}{c}\text { Decrease } \\
\text { (inches) }\end{array}$ & $\begin{array}{c}\text { Before } \\
\text { (inches) }\end{array}$ & $\begin{array}{c}\text { After } \\
\text { (inches) }\end{array}$ & $\begin{array}{l}\text { Decrease } \\
\text { (inches) }\end{array}$ \\
\hline \multirow[t]{4}{*}{10} & Rough & $\mathrm{D}_{\mathrm{m}}$ & 8.2 & 7.1 & 1.1 & 11.8 & 7.8 & 4.0 & 10.8 & 8.8 & 2.0 & 9.9 & 8.1 & 1.8 \\
\hline & & $\mathrm{S}_{\mathrm{m}}$ & 3.75 & 3.21 & 0.54 & 6.00 & 3.85 & 2.15 & 6.01 & 4.49 & 1.52 & 5.13 & 4.22 & 0.91 \\
\hline & Medium & $\mathrm{D}_{\mathrm{m}}$ & 8.0 & 6.3 & 1.7 & 7.5 & 7.0 & 0.5 & 8.8 & 6.8 & 2.0 & 7.5 & 6.4 & 1.1 \\
\hline & & $\mathrm{S}_{\mathrm{m}}$ & 4.29 & 3.00 & 1.29 & 3.25 & 2.90 & 0.35 & 4.19 & 2.91 & 1.28 & 3.47 & 3.17 & 0.30 \\
\hline \multirow[t]{4}{*}{30} & Rough & $\mathrm{D}_{\mathrm{m}}$ & 9.2 & 7.1 & 2.1 & 10.7 & 7.3 & 3.4 & 9.6 & 6.5 & 3.1 & 10.1 & 7.1 & 3.0 \\
\hline & & $\mathrm{S}_{\mathrm{m}}$ & 5.24 & 3.24 & 2.00 & 5.76 & 3.50 & 2.26 & 5.65 & 3.22 & 2.43 & 4.62 & 3.90 & 0.72 \\
\hline & Medium & $\mathrm{D}_{\mathrm{m}}$ & 8.7 & 7.8 & 0.9 & 9.2 & 7.1 & 2.1 & 6.9 & 6.4 & 0.5 & 9.7 & 6.1 & 3.6 \\
\hline & & $\mathrm{s}_{\mathrm{m}}$ & 4.33 & 3.79 & 0.54 & 5.29 & 3.82 & 1.47 & 3.34 & 2.82 & 0.52 & 4.98 & 3.07 & 1.91 \\
\hline \multirow[t]{4}{*}{50} & Rough & $\mathrm{D}_{\mathrm{m}}$ & 8.0 & 7.5 & 0.5 & 10.1 & 6.5 & 3.6 & 8.7 & 6.2 & 2.5 & 10.3 & 7.6 & 2.7 \\
\hline & & $\mathrm{S}_{\mathrm{m}}$ & 3.89 & 3.43 & 0.46 & 4.84 & 3.09 & 1.75 & 3.83 & 2.79 & 1.04 & 5.12 & 3.65 & 1.47 \\
\hline & Medium & $\mathrm{D}_{\mathrm{m}}$ & 7.2 & 5.9 & 1.3 & 6.3 & 5.8 & 0.5 & 8.0 & 7.2 & 0.8 & 6.8 & 4.9 & 1.9 \\
\hline & & $\mathrm{S}_{\mathrm{m}}$ & 3.28 & 2.68 & 0.60 & 3.09 & 3.06 & 0.03 & 4.55 & 4.17 & 0.38 & 3.10 & 1.77 & 1.33 \\
\hline
\end{tabular}


TABLE 4. Micro-Relief Maximum Depth and Storage Before and After Rainfall: Sparta Loamy Sand.

\begin{tabular}{|c|c|c|c|c|c|c|c|c|c|c|c|c|c|c|}
\hline \multirow{3}{*}{$\begin{array}{l}\text { Rainfall } \\
\text { Duration } \\
\text { (Min.) }\end{array}$} & \multirow[b]{3}{*}{$\begin{array}{l}\text { Surface } \\
\text { Condition }\end{array}$} & \multirow{3}{*}{$\begin{array}{l}\text { Maximum } \\
\text { Depth, } \mathrm{D}_{\mathrm{m}} \text {, } \\
\text { or Max. } \\
\text { Storage, } \mathrm{S}_{\mathrm{m}}\end{array}$} & \multicolumn{12}{|c|}{ Approximate Rainfall Intensity, iph } \\
\hline & & & \multicolumn{3}{|c|}{3} & \multicolumn{3}{|c|}{6} & \multicolumn{3}{|c|}{9} & \multicolumn{3}{|c|}{12} \\
\hline & & & $\begin{array}{l}\text { Before } \\
\text { (inches) }\end{array}$ & $\begin{array}{c}\text { After } \\
\text { (inches) }\end{array}$ & $\begin{array}{l}\text { Decrease } \\
\text { (inches) }\end{array}$ & $\begin{array}{l}\text { Before } \\
\text { (inches) }\end{array}$ & $\begin{array}{c}\text { After } \\
\text { (inches) }\end{array}$ & $\begin{array}{l}\text { Decrease } \\
\text { (inches) }\end{array}$ & $\begin{array}{l}\text { Before } \\
\text { (inches) }\end{array}$ & $\begin{array}{c}\text { After } \\
\text { (inches) }\end{array}$ & $\begin{array}{l}\text { Decrease } \\
\text { (inches) }\end{array}$ & $\begin{array}{l}\text { Before } \\
\text { (inches) }\end{array}$ & $\begin{array}{c}\text { After } \\
\text { (inches) }\end{array}$ & $\begin{array}{c}\text { Decrease } \\
\text { (inches) }\end{array}$ \\
\hline \multirow[t]{4}{*}{10} & Rough & $\mathrm{D}_{\mathrm{m}}$ & 6.7 & 5.8 & 0.9 & 6.6 & 4.9 & 1.7 & 6.8 & 4.5 & 2.3 & 7.5 & 5.3 & 2.2 \\
\hline & & $\mathrm{S}_{\mathrm{m}}$ & 3.24 & 2.75 & 0.49 & 2.72 & 1.86 & 0.86 & 2.81 & 1.92 & 0.89 & 3.01 & 2.31 & 0.70 \\
\hline & Medium & $\mathrm{D}_{\mathrm{m}}$ & 5.4 & 4.5 & 0.9 & 4.8 & 3.6 & 1.2 & 5.6 & 3.5 & 2.1 & 4.5 & 3.3 & 1.2 \\
\hline & & $\mathrm{S}_{\mathrm{m}}$ & 2.49 & 1.94 & 0.55 & 2.28 & 1.54 & 0.74 & 2.56 & 1.62 & 0.94 & 1.94 & 1.41 & 0.53 \\
\hline \multirow[t]{4}{*}{20} & Rough & $\mathrm{D}_{\mathrm{m}}$ & & & & & & & 6.2 & 4.3 & 1.9 & & & \\
\hline & & $\mathrm{S}_{\mathrm{m}}$ & & & & & & & 2.68 & 1.83 & 0.85 & & & \\
\hline & Medium & $\mathrm{D}_{\mathrm{m}}$ & & & & & & & 5.5 & 3.5 & 2.0 & & & \\
\hline & & $\mathrm{S}_{\mathrm{m}}$ & & & & & & & 2.36 & 1.40 & 0.96 & & & \\
\hline \multirow[t]{4}{*}{30} & Rough & $\mathrm{D}_{\mathrm{m}}$ & 6.1 & 4.4 & 1.7 & 7.9 & 6.7 & 1.2 & 6.5 & 3.4 & 3.1 & & & \\
\hline & & $\mathrm{S}_{\mathrm{m}}$ & 2.90 & 1.92 & 0.98 & 4.37 & 4.36 & 0.01 & 2.82 & 1.59 & 1.23 & & & \\
\hline & Medium & $\mathrm{D}_{\mathrm{m}}$ & 5.2 & 3.6 & 1.6 & 6.2 & 4.6 & 1.6 & 5.2 & 2.8 & 2.4 & & & \\
\hline & & $\mathrm{S}_{\mathrm{m}}$ & 2.49 & 1.57 & 0.92 & 2.91 & 1.99 & 0.92 & 2.36 & 0.79 & 1.57 & & & \\
\hline \multirow[t]{4}{*}{50} & Rough & $\mathrm{D}_{\mathrm{m}}$ & 6.9 & 5.6 & 1.3 & 7.0 & 3.7 & 3.3 & & & & & & \\
\hline & & $\mathrm{S}_{\mathrm{m}}$ & 3.05 & 1.90 & 1.15 & 3.94 & 1.43 & 2.51 & & & & & & \\
\hline & Medium & $\mathrm{D}_{\mathrm{m}}$ & 4.5 & 2.9 & 1.6 & 4.4 & 3.5 & 0.9 & & & & & & \\
\hline & & $\mathrm{S}_{\mathrm{m}}$ & 1.96 & 1.06 & 0.90 & 2.28 & 1.41 & 0.87 & & & & & & \\
\hline
\end{tabular}


TABLE 5. Micro-Relief Maximum Depth and Storage Before and After Rainfall: Darwin Silty Clay.

\begin{tabular}{|c|c|c|c|c|c|c|c|c|c|c|c|c|c|c|}
\hline \multirow{3}{*}{$\begin{array}{c}\text { Rainfall } \\
\text { Duration } \\
\text { (Min.) }\end{array}$} & \multirow[b]{3}{*}{$\begin{array}{l}\text { Surface } \\
\text { Condition }\end{array}$} & \multirow{3}{*}{$\begin{array}{l}\text { Maximum } \\
\text { Depth, } \mathrm{D}_{\mathrm{m}} \text {, } \\
\text { or Max. } \\
\text { Storage, } \mathrm{S}_{\mathrm{m}}\end{array}$} & \multicolumn{12}{|c|}{ Approximate Rainfall Intensity, iph } \\
\hline & & & \multicolumn{3}{|c|}{3} & \multicolumn{3}{|c|}{6} & \multicolumn{3}{|c|}{9} & \multicolumn{3}{|c|}{12} \\
\hline & & & $\begin{array}{c}\text { Before } \\
\text { (inches) }\end{array}$ & $\begin{array}{c}\text { After } \\
\text { (inches) }\end{array}$ & $\begin{array}{l}\text { Decrease } \\
\text { (inches) }\end{array}$ & $\begin{array}{l}\text { Before } \\
\text { (inches) }\end{array}$ & $\begin{array}{c}\text { After } \\
\text { (inches) }\end{array}$ & $\begin{array}{c}\text { Decrease } \\
\text { (inches) }\end{array}$ & $\begin{array}{l}\text { Before } \\
\text { (inches) }\end{array}$ & $\begin{array}{c}\text { After } \\
\text { (inches) }\end{array}$ & $\begin{array}{c}\text { Decrease } \\
\text { (inches) }\end{array}$ & $\begin{array}{l}\text { Before } \\
\text { (inches) }\end{array}$ & $\begin{array}{c}\text { After } \\
\text { (inches) }\end{array}$ & $\begin{array}{c}\text { Decrease } \\
\text { (inches) }\end{array}$ \\
\hline \multirow[t]{4}{*}{10} & Rough & $\mathrm{D}_{\mathrm{m}}$ & 13.5 & 13.1 & 0.4 & 11.5 & 11.1 & 0.4 & 12.8 & 11.9 & 0.9 & 12.6 & 10.6 & 2.0 \\
\hline & & $\mathrm{S}_{\mathrm{m}}^{\mathrm{M}}$ & 7.30 & 7.11 & 0.19 & 5.79 & 5.57 & 0.22 & 7.19 & 6.56 & 0.63 & 5.17 & 6.04 & 0.87 \\
\hline & Medium & $\mathrm{D}_{\mathrm{m}}$ & 10.9 & 9.7 & 1.2 & 11.4 & 9.5 & 1.9 & 11.5 & 11.2 & 0.3 & 11.3 & 11.0 & 0.3 \\
\hline & & $\mathrm{s}_{\mathrm{m}}$ & 6.01 & 4.72 & 1.29 & 5.46 & 4.86 & 0.60 & 5.96 & 5.56 & 0.40 & 5.43 & 5.42 & 0.01 \\
\hline \multirow[t]{4}{*}{30} & Rough & $\mathrm{D}_{\mathrm{m}}$ & 12.9 & 12.5 & 0.4 & 11.5 & 11.3 & 0.2 & 12.6 & 12.5 & 0.1 & 11.6 & 11.5 & 0.1 \\
\hline & & $\mathrm{s}_{\mathrm{m}}$ & 7.49 & 7.43 & 0.06 & 6.16 & 6.07 & 0.09 & 7.34 & 7.49 & -0.15 & 6.01 & 5.83 & 0.18 \\
\hline & Medium & $\mathrm{D}_{\mathrm{m}}$ & 11.3 & 10.8 & 0.5 & 11.1 & 10.1 & 1.0 & 11.8 & 11.2 & 0.6 & 10.5 & 10.0 & 0.5 \\
\hline & & $\mathrm{s}_{\mathrm{m}}$ & 6.08 & 5.70 & 0.38 & 6.74 & 5.71 & 1.03 & 6.13 & 5.37 & 0.76 & 5.11 & 4.69 & 0.42 \\
\hline \multirow[t]{4}{*}{50} & Rough & $\mathrm{D}_{\mathrm{m}}$ & 12.5 & 12.1 & 0.4 & 13.1 & 12.4 & 0.7 & 11.6 & 11.1 & 0.5 & 12.5 & 12.2 & 0.3 \\
\hline & & $\mathrm{s}_{\mathrm{m}}$ & 7.43 & 7.31 & 0.12 & 6.77 & 6.33 & 0.44 & 6.12 & 5.83 & 0.29 & 7.31 & 7.36 & -0.05 \\
\hline & Medium & $\mathrm{D}_{\mathrm{m}}$ & 9.8 & 9.3 & 0.5 & 10.6 & 9.8 & 0.8 & 10.7 & 9.9 & 0.8 & 11.3 & 11.4 & 0.1 \\
\hline & & $\mathrm{S}_{\mathrm{m}}$ & 5.55 & 5.03 & 0.52 & 6.04 & 5.55 & 0.49 & 5.60 & 4.79 & 0.81 & 6.07 & 5.97 & 0.10 \\
\hline
\end{tabular}


for a rough surface relief. In all but one case, the maximum depth decreased during the rainfall event. as would be expected. Except for thiee cases on Darwin silty clay soil. the maximum storage decreased during the rainfall event. The exceptions result from a change in the nature of the surface because of the rainfall event or are a result of the soil measurement variability.

Multiple regression analyses were performed to determine empirical relationships of the parameters effecting changes in the soil surface configuration during a rainfall event.

The variables considered were as follows:

1. $\Delta \mathrm{D} / \mathrm{D}_{\mathrm{O}}$, the change in maximum soil surface depth divided by the initial maximum soil surface depth.

2. $\Delta \mathrm{S}_{\mathrm{S}} / \mathrm{S}_{\mathrm{O}}$. the change in maximum surface depression storage divided by the initial maximum surface depression storage.

3. $\triangle \mathrm{a} / \mathrm{a}_{\mathrm{O}}$, the change in parameter a of Equation (2) divided by the initial value of parameter a.

4. $\Delta b / b_{0}$, the change in parameter $\underline{b}$ of Equation (2) divided by the initial value of the parameter $b$.

5 . I. the rainfall intensity in inches per hour.

6 . T. the rainfall duration in hours.

7. $Q_{R}$ and $Q_{P}$, the peak rates of runoff and percolation, respectively, during the rainfall event in inches per hour.

8. $V_{R} \cdot V_{I}$, and $V_{P}$, the volumes of runoff, infiltration and percolation, respectively, during the rainfall event in inches.

9. $\mathrm{M}_{\mathrm{O}}$, the initial soil moisture content in percent (dry basis).

10. $\mathrm{P}_{\mathrm{Sa}}$ and $\mathrm{P}_{\mathrm{cl}}$. the percent sand and clay size particles, respectively, in the soil tested.

A stepwise regression procedure was used to determine the relative importance of each term. Several different equation forms - linear, logarithmic, and polynomial - were attempted in the analysis. Early attempts showed that the logarithmic terms gave no better relationships than the linear relationships, when both ease of use and correlation coefficients were considered. Some polynomial terms and some cross product terms of the independent variables provided the best fit to the change in soil configuration data.

The results of the stepwise regression fit of the terms listed above are shown in Tables 6 through 10. The equations are the best fit prediction equations describing the change in maximum depth, maximum depression storage and the depth-storage model parameters $\underline{a}$ and $\underline{b}$. Tables 6 through 9 give the prediction equations for each of the four soils tested while Table 10 contains the prediction equations for all the soil test runs analyzed together. The multiple correlation coefficient. $R$, and the level of significance is indicated for each equation.

Five of the ten steps of the stepwise regression analysis are shown for each dependent variable. A greater number of terms in the prediction equation provides a statistically better fit, but the equation soon becomes unwieldly and does not significantly improve the quality of fit.

An examination of the tables reveals that the same terms do not necessarily appear for the same dependent variable across soil types. For instance, for the equations for change in maximum depth, $\Delta \mathrm{D} / \mathrm{D}_{\mathrm{O}}$, the first term entered in Table 6 is $T \mathrm{~V}_{\mathrm{R}} \mathrm{V}_{\mathrm{I}}$ and in Table 9 it is $\mathrm{M}_{\mathrm{O}}{ }^{2}$. None of the terms are identical between soils equations for the change in maximum depth dependent variable. A similar situation exists for the other dependent 
TABLE 6. Flanagan Silt Loam Prediction Equations.

\begin{tabular}{|c|c|c|}
\hline $\begin{array}{c}\text { Equation } \\
\text { Number }\end{array}$ & Prediction Equation & $\begin{array}{l}\text { Mult. Corr. } \\
\text { Coeff., R* }\end{array}$ \\
\hline $\begin{array}{l}4 \\
5 \\
6 \\
7 \\
8\end{array}$ & 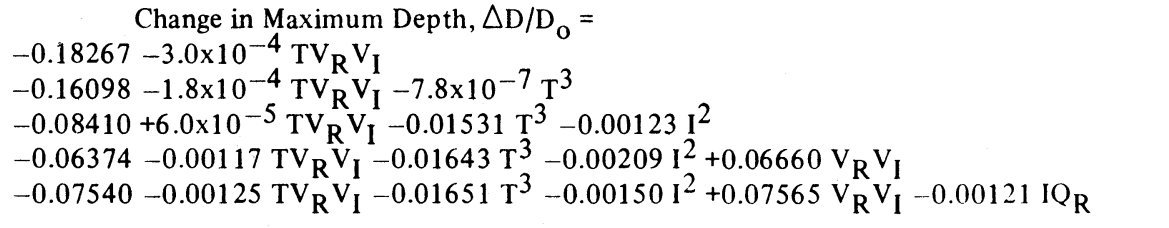 & $\begin{array}{l}0.653 \\
0.707 \\
0.810 \\
0.883 \\
0.889\end{array}$ \\
\hline $\begin{array}{r}9 \\
10 \\
11 \\
12 \\
13\end{array}$ & $\begin{array}{l}\text { Change in Maximum Storage, } \Delta \mathrm{S} / \mathrm{S}_{\mathrm{o}}= \\
-0.08045-9.0 \times 10^{-4} \mathrm{TV}_{\mathrm{I}} \\
-0.07981-5.5 \times 10^{-4} \mathrm{TV}_{\mathrm{I}}-5.0 \times 10^{-8} \mathrm{~T}^{3} \\
-0.12994-9.0 \times 10^{-4} \mathrm{TV}_{\mathrm{I}}-5.1 \times 10^{-7} \mathrm{~T}^{3}+2.7 \times 10^{-4} \mathrm{~T}^{2} \\
-0.11758-5.7 \times 10^{-4} \mathrm{TV}_{\mathrm{I}}-5.9 \times 10^{-7} \mathrm{~T}^{3}+3.1 \times 10^{-4} \mathrm{~T}^{2}-0.01943 \mathrm{Q}_{\mathrm{P}} \\
-0.09821-0.00161 \mathrm{TV}_{\mathrm{I}}-8.4 \times 10^{-7} \mathrm{~T}^{3}+4.4 \times 10^{-4} \mathrm{~T}^{2}-0.04352 \mathrm{Q}_{\mathrm{P}}+0.00266 \mathrm{TV}_{\mathrm{P}}\end{array}$ & $\begin{array}{l}0.668 \\
0.687 \\
0.785 \\
0.817 \\
0.882\end{array}$ \\
\hline $\begin{array}{l}14 \\
15 \\
16 \\
17 \\
18\end{array}$ & 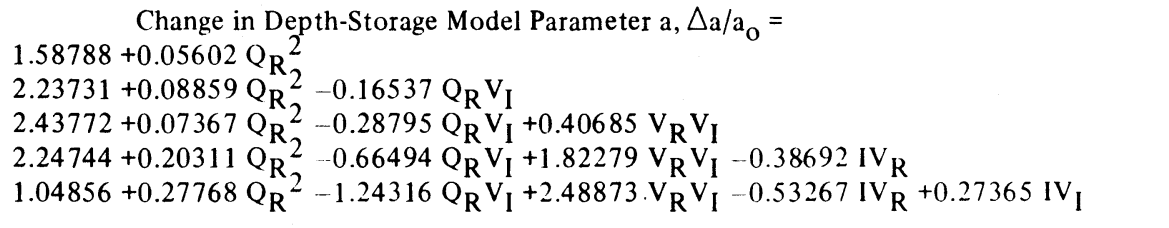 & $\begin{array}{l}0.681 \\
0.748 \\
0.778 \\
0.866 \\
0.900\end{array}$ \\
\hline $\begin{array}{l}19 \\
20 \\
21 \\
22 \\
23\end{array}$ & 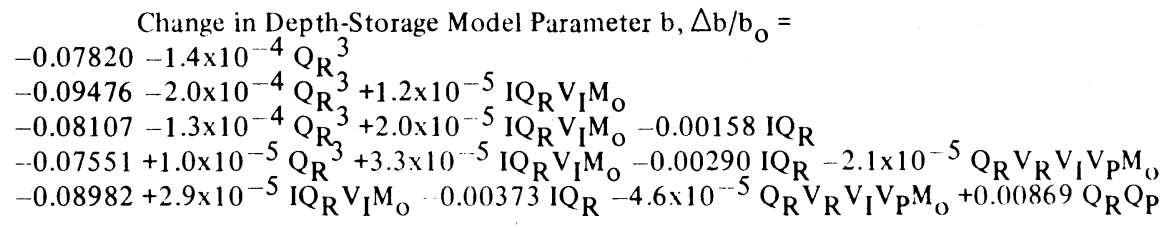 & $\begin{array}{l}0.675 \\
0.733 \\
0.758 \\
0.780 \\
0.814\end{array}$ \\
\hline
\end{tabular}

*All Equations significant at the $1 \%$ level. 
TABLE 7. Cisne Silt Loam Prediction Equations.

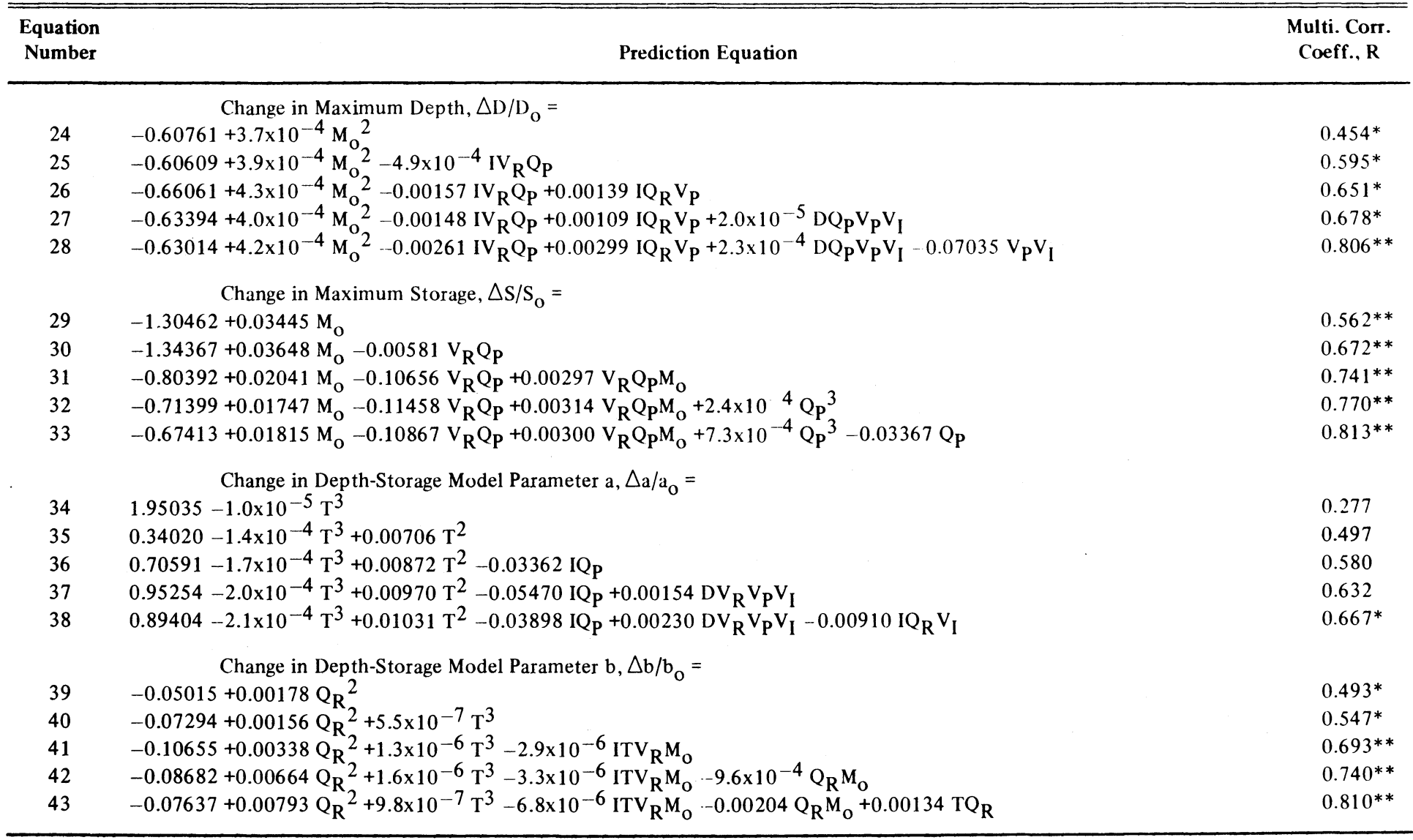

*Significant at the $5 \%$ level.

** Significant at the $1 \%$ level. 
TABLE 8. Sparta Loamy Sand Prediction Equations.

\begin{tabular}{|c|c|c|}
\hline $\begin{array}{c}\text { Equation } \\
\text { Number }\end{array}$ & Prediction Equation & $\begin{array}{l}\text { Multi. Corr. } \\
\text { Coeff., } R^{*}\end{array}$ \\
\hline \multicolumn{3}{|c|}{ Change in Maximum Depth, $\Delta D / D_{0}=$} \\
\hline 44 & $-0.23488-0.00560 \mathrm{IV}_{\mathrm{R}}$ & 0.637 \\
\hline 45 & $-0.25300-0.00614 \mathrm{IV}_{\mathrm{R}}+0.07139 \mathrm{Q}_{\mathrm{R}} \mathrm{V}_{\mathrm{P}}$ & 0.767 \\
\hline 46 & $-0.21856-0.00609 \mathrm{IV}_{\mathrm{R}}+0.13536 \mathrm{Q}_{\mathrm{R}} \mathrm{V}_{\mathrm{P}}-0.00203 \mathrm{TV}_{\mathrm{I}}$ & 0.874 \\
\hline 47 & $-0.21442-0.00532 \mathrm{IV}_{\mathrm{R}}+0.12416 \mathrm{Q}_{\mathrm{R}} \mathrm{V}_{\mathrm{P}}-0.00272 \mathrm{TV}_{\mathrm{I}}+0.25266 \mathrm{~V}_{\mathrm{P}}^{2}$ & 0.893 \\
\hline 48 & $-0.14194-0.00325 \mathrm{IV}_{\mathrm{R}}+0.12816 \mathrm{Q}_{\mathrm{R}} \mathrm{V}_{\mathrm{P}}-0.00383 \mathrm{TV}_{\mathrm{I}}+0.39414 \mathrm{~V}_{\mathrm{P}}^{2}-6.9 \times 10^{-4} \mathrm{IM}_{\mathrm{O}}$ & 0.921 \\
\hline \multicolumn{3}{|c|}{ Change in Maximum Storage, $\Delta S / S_{0}=$} \\
\hline 49 & $-0.09630-0.02261 \mathrm{I}$ & 0.588 \\
\hline 50 & $-0.12386-0.02106 \mathrm{I}+3.0 \times 10^{-5} \mathrm{ITV}_{\mathrm{R}} \mathrm{V}_{\mathrm{I}} \mathrm{V}_{\mathrm{P}} \mathrm{M}_{\mathrm{O}}$ & 0.717 \\
\hline 51 & $-0.14035-0.01725 \mathrm{I}+1.4 \times 10^{-4} \mathrm{ITV}_{\mathrm{R}} \mathrm{V}_{\mathrm{I}} \mathrm{V}_{\mathrm{P}} \mathrm{M}_{\mathrm{O}}-0.11305 \mathrm{Q}_{\mathrm{R}} \mathrm{V}_{\mathrm{R}} \mathrm{V}_{\mathrm{P}}$ & 0.780 \\
\hline 52 & $-0.15989-0.01650 \mathrm{I}+2.9 \times 10^{-4} \mathrm{ITV}_{\mathrm{R}} \mathrm{V}_{\mathrm{I}} \mathrm{V}_{\mathrm{P}} \mathrm{M}_{\mathrm{o}}-0.31452 \mathrm{Q}_{\mathrm{R}} \mathrm{V}_{\mathrm{R}} \mathrm{V}_{\mathrm{P}}+0.00107 \mathrm{Q}_{\mathrm{R}} \mathrm{Q}_{\mathrm{P}} \mathrm{V}_{\mathrm{R}} \mathrm{M}_{\mathrm{O}}$ & 0.829 \\
\hline 53 & $-0.04315-0.01898 \mathrm{I}+4.9 \times 10^{-4} \mathrm{ITV}_{\mathrm{R}} \mathrm{V}_{\mathrm{I}} \mathrm{V}_{\mathrm{P}} \mathrm{M}_{\mathrm{o}}-0.55613 \mathrm{Q}_{\mathrm{R}} \mathrm{V}_{\mathrm{R}} \mathrm{V}_{\mathrm{P}}+0.00219 \mathrm{Q}_{\mathrm{R}} \mathrm{Q}_{\mathrm{P}} \mathrm{V}_{\mathrm{R}} \mathrm{M}_{\mathrm{o}}-0.13581 \mathrm{~V}_{\mathrm{I}}$ & 0.920 \\
\hline \multicolumn{3}{|c|}{ Change in Depth-Storage Model Parameter $\mathrm{a}, \Delta \mathrm{a} / \mathrm{a}_{\mathrm{o}}=$} \\
\hline 54 & $-0.23027+0.03348 \mathrm{TV}_{\mathrm{R}} \mathrm{V}_{\mathrm{I}}$ & 0.624 \\
\hline 55 & $-0.47582+0.06717 \mathrm{TV}_{\mathrm{R}} \mathrm{V}_{\mathrm{I}}-0.29811 \mathrm{IQ}_{\mathrm{R}} \mathrm{V}_{\mathrm{R}} \mathrm{V}_{\mathrm{P}}$ & 0.892 \\
\hline 56 & $0.06951+0.10107 \mathrm{TV}_{\mathrm{R}} \mathrm{V}_{\mathrm{I}}-0.20222 \mathrm{IQ}_{\mathrm{R}} \mathrm{V}_{\mathrm{R}} \mathrm{V}_{\mathrm{P}}-0.00427 \mathrm{TV}_{\mathrm{R}} \mathrm{M}_{\mathrm{O}}$ & 0.925 \\
\hline 57 & $0.73672+0.14306 \mathrm{TV}_{\mathrm{R}} \mathrm{V}_{\mathrm{I}}+0.32987 \mathrm{IQ}_{\mathrm{R}} \mathrm{V}_{\mathrm{R}} \mathrm{V}_{\mathrm{P}}-0.00959 \mathrm{TV}_{\mathrm{R}} \mathrm{M}_{\mathrm{o}}-0.34757 \mathrm{TQ}_{\mathrm{R}} \mathrm{V}_{\mathrm{R}} \mathrm{M}_{0}$ & 0.971 \\
\hline 58 & $0.88704+0.14260 \mathrm{TV}_{\mathrm{R}} \mathrm{V}_{\mathrm{I}}+0.38974 \mathrm{IQ}_{\mathrm{R}} \mathrm{V}_{\mathrm{R}} \mathrm{V}_{\mathrm{P}}-0.00731 \mathrm{TV}_{\mathrm{R}} \mathrm{M}_{\mathrm{o}}-0.44206 \mathrm{TQ}_{\mathrm{R}} \mathrm{V}_{\mathrm{R}^{\prime}} \mathrm{M}_{\mathrm{o}}-0.12350 \mathrm{Q}_{\mathrm{R}} \mathrm{V}_{\mathrm{R}} \mathrm{V}_{\mathrm{I}}$ & 0.978 \\
\hline \multicolumn{3}{|c|}{ Change in Depth-Storage Model Parameter $b, \Delta b / b_{0}=$} \\
\hline 59 & $-0.53316+0.03880 \mathrm{M}_{\mathrm{o}}$ & 0.565 \\
\hline 60 & $-0.58740+0.03507 \mathrm{M}_{\mathrm{o}}+0.01615 \mathrm{I}$ & 0.622 \\
\hline 61 & $-0.57022+0.03227 \mathrm{M}_{\mathrm{o}}+0.01718 \mathrm{I}+0.00196 \mathrm{~V}_{\mathrm{R}} \mathrm{Q}_{\mathrm{P}} \mathrm{M}_{\mathrm{O}}$ & 0.643 \\
\hline 62 & $-0.23921+0.00301 \mathrm{M}_{\mathrm{o}}+0.03075 \mathrm{I}+0.02486 \mathrm{~V}_{\mathrm{R}} \mathrm{Q}_{\mathrm{P}} \mathrm{M}_{\mathrm{o}}-0.00130 \mathrm{TQ}_{\mathrm{R}} \mathrm{V}_{\mathrm{R}} \mathrm{Q}_{\mathrm{P}}$ & 0.913 \\
\hline 63 & $-0.10481-0.00575 \mathrm{M}_{\mathrm{o}}+0.03242 \mathrm{I}+0.02445 \mathrm{~V}_{\mathrm{R}} \mathrm{Q}_{\mathrm{P}} \mathrm{M}_{\mathrm{o}}-0.00187 \mathrm{TQ}_{\mathrm{R}} \mathrm{V}_{\mathrm{R}} \mathrm{Q}_{\mathrm{P}}+0.01579 \mathrm{IQ}_{\mathrm{R}} \mathrm{V}_{\mathrm{R}} \mathrm{V}_{\mathrm{P}}$ & 0.946 \\
\hline
\end{tabular}

*All Equations significant at the $1 \%$ level. 
TABLE 9. Darwin Silty Clay Prediction Equations.

\begin{tabular}{|c|c|c|}
\hline $\begin{array}{l}\text { Equation } \\
\text { Number }\end{array}$ & Prediction Equation & $\begin{array}{l}\text { Multi. Corr. } \\
\text { Coeff., R }\end{array}$ \\
\hline & Change in Maximum Depth, $\Delta \mathrm{D} / \mathrm{D}_{\mathrm{o}}=$ & \\
\hline 64 & $-0.07321+0.02059 \mathrm{~V}_{\mathrm{P}}$ & $0.471^{*}$ \\
\hline 65 & $-0.27306+0.19543 \mathrm{~V}_{\mathrm{P}}+0.00579 \mathrm{M}_{\mathrm{o}}$ & $0.628 * *$ \\
\hline 66 & $-0.30909+0.02445 \mathrm{~V}_{\mathrm{P}}+0.00688 \mathrm{M}_{\mathrm{o}}+0.05133 \mathrm{IQ}_{\mathrm{P}} \mathrm{V}_{\mathrm{P}}$ & $0.673 * *$ \\
\hline 67 & $-0.37407-0.09198 \mathrm{~V}_{\mathrm{P}}+0.00905 \mathrm{M}_{\mathrm{o}}+0.12608 \mathrm{IQ}_{\mathrm{P}} \mathrm{V}_{\mathrm{P}}-3.8 \times 10^{-4} \mathrm{Q}_{\mathrm{R}} \mathrm{Q}_{\mathrm{P}} \mathrm{M}_{\mathrm{o}}$ & $0.765^{* *}$ \\
\hline \multirow[t]{2}{*}{68} & $-0.39186-0.19942 \mathrm{~V}_{\mathrm{P}}+0.00946 \mathrm{M}_{\mathrm{o}}+0.30847 \mathrm{IQ}_{\mathrm{P}} \mathrm{V}_{\mathrm{P}}-7.1 \times 10^{-4} \mathrm{Q}_{\mathrm{R}} \mathrm{Q}_{\mathrm{P}} \mathrm{M}_{\mathrm{o}}-0.00120 \mathrm{TV}_{\mathrm{R}} \mathrm{V}_{\mathrm{I}} \mathrm{V}_{\mathrm{P}}$ & $0.863^{* *}$ \\
\hline & Change in Maximum Storage, $\Delta \mathrm{S} / \mathrm{S}_{\mathrm{o}}=$ & \\
\hline 69 & $-0.09170+0.00155 \mathrm{~T}$ & 0.292 \\
\hline 70 & $-0.07994+0.00199 \mathrm{~T}-4.8 \times 10^{-5} \mathrm{Q}_{\mathrm{R}}^{3}$ & 0.459 \\
\hline 71 & $-0.05942-0.00137 \mathrm{~T}-1.3 \times 10^{-4} \mathrm{Q}_{\mathrm{R}}^{3}+1.6 \times 10^{-5} \mathrm{ITM}_{\mathrm{O}}$ & $0.668^{* *}$ \\
\hline 72 & $-0.11792-0.00166 \mathrm{~T}-1.6 \times 10^{-4} \mathrm{Q}_{\mathrm{R}}^{3}+3.8 \times 10^{-5} \mathrm{ITM}_{\mathrm{O}}-2.8 \times 10^{-5} \mathrm{TV}_{\mathrm{R}} \mathrm{M}_{\mathrm{O}}$ & $0.745^{* *}$ \\
\hline \multirow[t]{2}{*}{73} & $-0.11749+3.9 \times 10^{-4} \mathrm{~T}-1.9 \times 10^{-4} \mathrm{Q}_{\mathrm{R}}^{3}+2.9 \times 10^{-5} \mathrm{ITM}_{\mathrm{o}}-4.4 \times 10^{-5} \mathrm{TV}_{\mathrm{R}} \mathrm{M}_{\mathrm{o}}+3.5 \times 10^{-4} \mathrm{IQ}_{\mathrm{R}} \mathrm{V}_{\mathrm{R}}$ & $0.807^{* *}$ \\
\hline & Change in Depth-Storage Model Parameter $\mathrm{a}, \Delta \mathrm{a} / \mathrm{a}_{\mathrm{o}}=$ & \\
\hline 74 & $0.88744-0.19628 \mathrm{IQ}_{\mathrm{P}}$ & $0.543^{* *}$ \\
\hline 75 & $0.58848-0.25538 \mathrm{IQ}_{\mathrm{P}}+0.61259 \mathrm{~V}_{\mathrm{I}}$ & $0.661^{* *}$ \\
\hline 76 & $0.74794-0.30474 \mathrm{IQ}_{\mathrm{P}}+0.91315 \mathrm{~V}_{\mathrm{I}}-5.1 \times 10^{-6} \mathrm{~T}^{3}$ & $0.739 * *$ \\
\hline 77 & $0.45428-0.31532 \mathrm{IQ}_{\mathrm{P}}+1.89797 \mathrm{~V}_{\mathrm{I}}-5.5 \times 10^{-6} \mathrm{~T}^{3}-0.49131 \mathrm{~V}_{\mathrm{I}}^{2}$ & $0.777^{* *}$ \\
\hline \multirow[t]{2}{*}{78} & $2.18710-0.30439 \mathrm{IQ}_{\mathrm{P}}+2.11884 \mathrm{~V}_{\mathrm{I}}-5.0 \times 10^{-6} \mathrm{~T}^{3}-0.69027 \mathrm{~V}_{\mathrm{I}}^{2}-0.05173 \mathrm{M}_{\mathrm{o}}$ & $0.797 * *$ \\
\hline & Change in Depth-Storage Model Parameter $b, \Delta b / b_{o}=$ & \\
\hline 79 & $-0.05423+6.4 \times 10^{-4} \mathrm{Q}_{\mathrm{R}} \mathrm{Q}_{\mathrm{P}} \mathrm{M}_{\mathrm{o}}$ & $0.508^{*}$ \\
\hline 80 & $-0.04531+0.00151 \mathrm{Q}_{\mathrm{R}} \mathrm{Q}_{\mathrm{P}} \mathrm{M}_{\mathrm{o}}-3.0 \times 10^{-5} \mathrm{IQ}_{\mathrm{R}} \mathrm{V}_{\mathrm{I}} \mathrm{M}_{\mathrm{o}}$ & $0.704 * *$ \\
\hline 81 & $-0.04565+0.00115 \mathrm{Q}_{\mathrm{R}} \mathrm{Q}_{\mathrm{P}} \mathrm{M}_{\mathrm{o}}-5.0 \times 10^{-5} \mathrm{IQ}_{\mathrm{R}} \mathrm{V}_{\mathrm{I}} \mathrm{M}_{\mathrm{o}}+1.1 \times 10^{-4} \mathrm{Q}_{\mathrm{R}}^{3}$ & $0.799 * *$ \\
\hline 82 & $-0.04098+0.00126 \mathrm{Q}_{\mathrm{R}} \mathrm{Q}_{\mathrm{P}} \mathrm{M}_{\mathrm{o}}-9.0 \times 10^{-5} \mathrm{IQ}_{\mathrm{R}} \mathrm{V}_{\mathrm{I}} \mathrm{M}_{\mathrm{o}}+1.6 \times 10^{-4} \mathrm{Q}_{\mathrm{R}}^{3}+0.00121 \mathrm{TV}_{\mathrm{R}} \mathrm{V}_{\mathrm{I}} \mathrm{V}_{\mathrm{P}}$ & $0.863^{* *}$ \\
\hline 83 & $-0.05990+5.8 \times 10^{-4} \mathrm{Q}_{\mathrm{R}} \mathrm{Q}_{\mathrm{P}} \mathrm{M}_{\mathrm{o}}-9.0 \times 10^{-5} \mathrm{IQ}_{\mathrm{R}} \mathrm{V}_{\mathrm{I}} \mathrm{M}_{\mathrm{O}}+1.9 \times 10^{-4} \mathrm{Q}_{\mathrm{R}}^{3}+0.00125 \mathrm{TV}_{\mathrm{R}} \mathrm{V}_{\mathrm{I}} \mathrm{V}_{\mathrm{P}}+0.02047 \mathrm{IQ}_{\mathrm{P}}$ & $0.895^{* *}$ \\
\hline
\end{tabular}

* Significant at the $5 \%$ level.

** Significant at the $1 \%$ level. 
TABLE 10. All Soils Prediction Equations.

\begin{tabular}{|c|c|c|}
\hline $\begin{array}{l}\text { Equation } \\
\text { Number }\end{array}$ & Prediction Equation & $\begin{array}{l}\text { Multi. Corr. } \\
\text { Coeff., } R^{*}\end{array}$ \\
\hline \multicolumn{3}{|c|}{ Change in Maximum Depth, $\Delta \mathrm{D} / \mathrm{D}_{\mathrm{o}}=$} \\
\hline 84 & $-0.33004+1.7 \times 10^{-4} \mathrm{M}_{\mathrm{o}} \mathbf{P}_{\mathrm{cl}}$ & 0.611 \\
\hline 85 & $-0.31135+1.7 \times 10^{-4} \mathrm{M}_{\mathrm{o}} \mathrm{P}_{\mathrm{cl}}-0.00638 \mathrm{~V}_{\mathrm{R}} \mathrm{Q}_{\mathrm{P}}$ & 0.745 \\
\hline 86 & $-0.33081+1.8 \times 10^{-4} \mathrm{M}_{\mathrm{o}} \mathrm{P}_{\mathrm{cl}}-0.00895 \mathrm{~V}_{\mathrm{R}} \mathrm{Q}_{\mathrm{P}}+0.00188 \mathrm{~V}_{\mathrm{P}} \mathrm{P}_{\mathrm{sa}}$ & 0.764 \\
\hline 87 & $-0.30134+1.8 \times 10^{-4} \mathrm{M}_{\mathrm{o}} \mathrm{P}_{\mathrm{cl}}-0.00692 \mathrm{~V}_{\mathrm{R}} \mathrm{Q}_{\mathrm{P}}+0.00364 \mathrm{~V}_{\mathrm{P}} \mathrm{P}_{\mathrm{sa}}-0.04169 \mathrm{~V}_{\mathrm{I}}$ & 0.793 \\
\hline 88 & $-0.22733+1.0 \times 10^{-4} \mathrm{M}_{\mathrm{o}} \mathrm{P}_{\mathrm{cl}}-0.00697 \mathrm{~V}_{\mathrm{R}} \mathrm{Q}_{\mathrm{P}}+0.00665 \mathrm{~V}_{\mathrm{P}} \mathrm{P}_{\mathrm{sa}}-0.17746 \mathrm{~V}_{\mathrm{I}}+0.00419 \mathrm{~V}_{\mathrm{I}} \mathrm{P}_{\mathrm{cl}}$ & 0.816 \\
\hline \multicolumn{3}{|c|}{ Change in Maximum Storage, $\Delta \mathrm{S} / \mathrm{S}_{\mathrm{o}}=$} \\
\hline 89 & $-0.26722+1.5 \times 10^{-4} \mathrm{M}_{\mathrm{o}} \mathrm{P}_{\mathrm{cl}}$ & 0.602 \\
\hline 90 & $-0.19131+1.5 \times 10^{-4} \mathrm{M}_{\mathrm{o}} \mathrm{P}_{\mathrm{cl}}-0.01124 \mathrm{I}$ & 0.671 \\
\hline 91 & $-0.11705+7.0 \times 10^{-5} \mathrm{M}_{\mathrm{o}} \mathrm{P}_{\mathrm{cl}}-0.02429 \mathrm{I}+4.7 \times 10^{-4} \mathrm{IP}_{\mathrm{cl}}$ & 0.695 \\
\hline 92 & $-0.11516+7.0 \times 10^{-5} \mathrm{M}_{\mathrm{o}} \mathrm{P}_{\mathrm{cl}}-0.02334 \mathrm{I}+4.5 \times 10^{-4} \mathrm{IP}_{\mathrm{cl}}-1.7 \times 10^{-5} \mathrm{TV}_{\mathrm{P}} \mathrm{P}_{\mathrm{cl}}$ & 0.704 \\
\hline 93 & $-0.09665+6.0 \times 10^{-5} \mathrm{M}_{\mathrm{o}} \mathrm{P}_{\mathrm{cl}}-0.02685 \mathrm{I}+5.5 \times 10^{-4} \mathrm{IP}_{\mathrm{cl}}-5.1 \times 10^{-5} \mathrm{TV}_{\mathrm{P}} \mathrm{P}_{\mathrm{cl}}+4.0 \times 10^{-6} \mathrm{Q}_{\mathrm{P}} \mathrm{V}_{\mathrm{P}} \mathrm{V}_{\mathrm{I}} \mathrm{M}_{\mathrm{o}} \mathrm{P}_{\mathrm{sa}}$ & 0.734 \\
\hline \multicolumn{2}{|r|}{ Change in Depth-Storage Model Parameter $\mathrm{a}, \Delta \mathrm{a} / \mathrm{a}_{\mathrm{o}}=$} & 0.349 \\
\hline 94 & $1.20961+6.6 \times 10^{-4} \mathrm{Q}_{\mathrm{R}} \mathrm{V}_{\mathrm{R}} \mathrm{Q}_{\mathrm{P}} \mathrm{P}_{\mathrm{cl}}$ & 0.349 \\
\hline 95 & $1.28925+0.00891 \mathrm{Q}_{\mathrm{R}} \mathrm{V}_{\mathrm{R}} \mathrm{Q}_{\mathrm{P}} \mathrm{P}_{\mathrm{cl}}-2.6 \times 10^{-4} \mathrm{Q}_{\mathrm{R}} \mathrm{V}_{\mathrm{R}} \mathrm{Q}_{\mathrm{P}} \mathrm{M}_{\mathrm{o}} \mathrm{P}_{\mathrm{cl}}$ & 0.456 \\
\hline 96 & $0.98167+0.01014 \mathrm{Q}_{\mathrm{R}} \mathrm{V}_{\mathrm{R}} \mathrm{Q}_{\mathrm{P}} \mathrm{P}_{\mathrm{cl}}-3.1 \times 10^{-4} \mathrm{Q}_{\mathrm{R}} \mathrm{V}_{\mathrm{R}} \mathrm{Q}_{\mathrm{P}} \mathrm{M}_{\mathrm{o}} \mathrm{P}_{\mathrm{cl}}+1.6 \times 10^{-4} \mathrm{TV}_{\mathrm{R}} \mathrm{V}_{\mathrm{I}} \mathrm{P}_{\mathrm{sa}}$ & 0.500 \\
\hline 97 & $2.30350+0.00730 \mathrm{Q}_{\mathrm{R}} \mathrm{V}_{\mathrm{R}} \mathrm{Q}_{\mathrm{P}} \mathrm{P}_{\mathrm{cl}}-2.2 \times 10^{-4} \mathrm{Q}_{\mathrm{R}} \mathrm{V}_{\mathrm{R}} \mathrm{Q}_{\mathrm{P}} \mathrm{M}_{\mathrm{o}} \mathrm{P}_{\mathrm{cl}}+4.7 \times 10^{-4} \mathrm{TV}_{\mathrm{R}} \mathrm{V}_{\mathrm{I}} \mathrm{P}_{\mathrm{sa}}{ }^{\mathrm{a}}-1.5 \times 10^{-5} \mathrm{ITM}_{\mathrm{O}} \mathrm{P}_{\mathrm{sa}}$ & 0.607 \\
\hline 98 & $2.54551+0.00163 \mathrm{Q}_{\mathrm{R}} \mathrm{V}_{\mathrm{R}} \mathrm{Q}_{\mathrm{P}} \mathrm{P}_{\mathrm{cl}}-2.0 \times 10^{-5} \mathrm{Q}_{\mathrm{R}} \mathrm{V}_{\mathrm{R}} \mathrm{Q}_{\mathrm{P}} \mathrm{M}_{\mathrm{O}} \mathrm{P}_{\mathrm{cl}}+7.4 \times 10^{-4} \mathrm{TV}_{\mathrm{R}} \mathrm{V}_{\mathrm{I}} \mathrm{P}_{\mathrm{sa}}-1.9 \times 10^{-5} \mathrm{ITM}_{\mathrm{O}} \mathrm{P}_{\mathrm{sa}}-9.6 \times 10^{-4} \mathrm{TV}_{\mathrm{R}} \mathrm{V}_{\mathrm{P}} \mathrm{P}_{\mathrm{sa}}$ & 0.678 \\
\hline \multicolumn{3}{|c|}{ Change in Depth-Storage Model Parameter $b, \Delta b / b_{0}=$} \\
\hline 99 & $-0.01619+0.00147 \mathrm{Q}_{\mathrm{R}} \mathrm{M}_{\mathrm{o}} \mathrm{P}_{\mathrm{Sa}}$ & 0.819 \\
\hline 100 & $-0.05255+0.00147 \mathrm{Q}_{\mathrm{R}} \mathrm{M}_{\mathrm{O}} \mathrm{P}_{\mathrm{sa}}^{\mathrm{sa}}+0.00765 \mathrm{Q}_{\mathrm{R}}$ & 0.850 \\
\hline 101 & $-0.09919+0.00137 \mathrm{Q}_{\mathrm{R}} \mathrm{M}_{\mathrm{o}} \mathrm{P}_{\mathrm{sa}}+0.00657 \mathrm{Q}_{\mathrm{R}}+7.0 \times 10^{-5} \mathrm{M}_{\mathrm{o}} \mathrm{P}_{\mathrm{sa}}$ & 0.864 \\
\hline 102 & $-0.10171+0.00152 \mathrm{Q}_{\mathrm{R}} \mathrm{M}_{\mathrm{o}} \mathrm{P}_{\mathrm{sa}}+0.00415 \mathrm{Q}_{\mathrm{R}}+8.0 \times 10^{-5} \mathrm{M}_{\mathrm{o}} \mathrm{P}_{\mathrm{sa}}+1.6 \times 10^{-5} \mathrm{Q}_{\mathrm{R}} \mathrm{V}_{\mathrm{R}} \mathrm{Q}_{\mathrm{P}} \mathrm{P}_{\mathrm{cl}}$ & 0.875 \\
\hline 103 & $-0.11385+0.00156 \mathrm{Q}_{\mathrm{R}} \mathrm{M}_{\mathrm{o}} \mathrm{P}_{\mathrm{sa}}+0.01093 \mathrm{Q}_{\mathrm{R}}+8.0 \times 10^{-5} \mathrm{M}_{\mathrm{o}} \mathrm{P}_{\mathrm{sa}}+2.1 \times 10^{-5} \mathrm{Q}_{\mathrm{R}} \mathrm{V}_{\mathrm{R}} \mathrm{Q}_{\mathrm{P}} \mathrm{P}_{\mathrm{cl}}-6.2 \times 10^{-5} \mathrm{Q}_{\mathrm{R}}^{3}$ & 0.882 \\
\hline
\end{tabular}

*All Equations significant at the $1 \%$ level. 
variables between soils, except for the $\mathrm{Q}_{\mathrm{R}}{ }^{3}$ term between Flanagan and Darwin soils for the change in depth-storage model parameter $b$. Peak runoff rate. $Q_{R}$, and volume of runoff. $V_{R}$, are evident in many of the terms. indicating the importance of a runoff element for describing the changes. Rainfall intensity, I. and duration. T, are present singly or as a cross product in all soil equation groups: except for the change in maximum storage. $\Delta S / S_{0}$, equations for the Cisne soil.

An examination of the equation terms for a particular dependent variable across soil types (Tables 6 through 9) and for the pooled data (Table 10) reveals much the same situation discussed in the previous paragraph. The lack of agreement of terms between soils results from the attempts to create terms with the greatest statistical significance using many combinations of parameters. Each of these searches for the most significant terms was done first within a soil type and then with the data for all soils as a group. This resulted in equation terms that are characteristic of the reaction of that soil under the conditions tested.

Similarities of equation terms within a soil type are interesting, if not conclusive. For Flanagan silt loam (Table 6), rainfall duration, $T$, volume of infiltration, $\mathrm{V}_{\mathrm{I}}$, and peak rate of runoff, $Q_{R}$, seem important. For Cisne silt loam (Table 7), initial soil moisture, $\mathrm{M}_{\mathrm{O}}$, the $\mathrm{V}_{\mathrm{R}} \mathrm{Q}_{\mathrm{P}}$ term, and rainfall duration, $\mathrm{T}$, are most evident. The importance of $\mathrm{M}_{\mathrm{O}}$ to Cisne silt loam, as compared to Flanagan silt loam, may be because Cisne has low organic matter which causes it to crust. The crusting tendency and the percent clay difference interact to cause different terms to be important although Flanagan and Cisne are both silt loams. Rainfall intensity, $I$, and duration, $T$; peak rate, $Q_{R}$, and volume, $V_{R}$, of runoff; and initial soil moisture, $\mathrm{M}_{\mathrm{O}}$, are evident in the equations for all dependent variables for the Sparta loamy sand (Table 8 ). The variables $M_{O}, I$, and $T$ are evident in the last equations for all dependent variables for Darwin silty clay (Table 9), although no term seems consistently important in the earlier stage of the development of the equations.

For the pooled data equations (Table 10 ), initial soil moisture, $\mathrm{M}_{\mathrm{O}}$, and percent clay size soil particles are apparently important for all four dependent variables. The lack of either rainfall variable $\mathrm{I}$ or $\mathrm{T}$ in the change in maximum depth equation $\Delta \mathrm{D} / \mathrm{D}_{\mathrm{O}}$, and in the change in depth-storage model parameter $\underline{b}$ equation, $\Delta \mathrm{b} / \mathrm{b}_{\mathrm{O}}$, is disconcerting at best.

In many equations terms are present that indicate a relationship near to what one might expect. Fifty-eight equations have terms containing rainfall intensity, I, or duration, $\mathrm{T}$, singly or in combination with one other parameter. The peak rate of runoff parameter, $Q_{R}$, is evident in twenty-five of the equations as a single term and in several other equations in combination with other parameters. A similar situation may be observed with the other parameters used singly as equation terms and used as cross products with other parameters to provide equation terms.

Several equation terms are presented, however, that are difficult to relate to physical phenomena, especially those terms that are cross products of several parameters. This results, of course, from the statistical treatment of the prediction equation development. A few equations are presented that one may find difficult to understand, for instance Equations (24), (29), and (59), which have only the initial soil moisture term, $\mathrm{M}_{\mathrm{O}}$, and Equations (84) and (89), which have a cross product term of soil moisture and percent clay particles in the soil. Again, this results from the statistical treatment, and one should be wary of using statistically developed prediction equations beyond the limits of the conditions under which they were developed. These soils have inherent differences in the 
Mitchell and Jones, Jr.

way in which the micro-relief responds to the rainfall event. These differences are reflected in the equations selected as being the most significant.

\section{APPLICATION TO WATERSHED MODELS}

The relationships described in Tables 6 through 10 may be used in computer watershed models that iterate in successive time increments. Such models update the values of rainfall intensity and duration; soil moisture; and runoff, infiltration, and percolation volumes and rates for each time increment. The micro-relief surface depression storage can also be changed and evaluated for each time increment.

Any method of including micro-relief surface depression storage values in a computer model requires an estimate of the initial value for maximum soil surface depth, $\mathrm{D}_{\mathrm{O}}$, and, if deemed desirable, an estimate of the initial maximum micro-relief surface depression storage, $\mathrm{S}_{\mathrm{O}}$. Equations similar to those presented by Mitchell and Jones (1976), which use the initial values for $D$ as independent variables can be used to estimate the initial values for the depth-storage curve parameters $\underline{a}$ and $\underline{b}$ in Equation (2). From the initial value of the maximum depth, the depth-storage relationship is then defined.

From the initial value of the maximum depth and the initial values of the depthstorage function parameters $\underline{a}$ and $\underline{b}$, the initial value of surface depression storage can be determined. The actual amount of the depression storage used will depend upon the rainfall characteristics and the infiltration that occurs during the time increment used in the model. If only a part of the available depression storage is used, the rest is available for the next time increment. Runoff will begin when the available storage is exceeded.

The equations in Tables 6 through 10 can be used to estimate changes in micro-relief in computer watershed models. The particular table from which equations are selected will depend upon whether the equations for a particular soil are desired (Tables 6 through 9) or the modeler wishes to use the equations obtained from the pooled data (Table 10). Five equations are presented in each table for each of the four dependent variables. The particular equation selected to estimate the change in a micro-relief variable depends on the significance level desired and on the other hydrologic elements evaluated within the computer model. The change in micro-relief variable equations use other hydrologic data as independent variables; so those hydrologic elements must be evaluated in the watershed model if they are to be used in the equations defining the change in micro-relief. For instance, if peak percolation rate, $Q_{p}$, is not determined in the watershed model, then an equation that includes $Q_{P}$ as an independent variable cannot be used.

In a watershed model several hydrologic phenomena, including the micro-relief, can be evaluated for each time increment. If a variable, such as the change in maximum depth, $\triangle D / D_{0}$, requires the value of another phenomenon, that phenomenon may need to be estimated and an iterative procedure used to determine the final values.

At the end of each time increment, any one of several methods can be used to estimate the new surface depression function. In one approach the equations selected from Tables 6 through 10 could be used to estimate the changes expected in maximum depth and in parameters $\underline{a}$ and $\underline{b}$ of Equation (2): $\Delta \mathrm{D} / \mathrm{D}_{\mathrm{O}}, \Delta \mathrm{a} / \mathrm{a}_{\mathrm{O}}$, and $\Delta \mathrm{b} / \mathrm{b}_{\mathrm{O}}$, respectively. The changes multiplied by the initial values and added algebraically to the initial values would then provide the maximum depth and depth-storage curve parameter values of $\mathrm{D}_{1}, \mathrm{a}_{1}$, and $b_{1}$ that would be applicable at the end of the first time increment or at the start of the second time increment. 
Another approach would be to determine only the change in depth or perhaps the change in depth and the change in one depth-storage curve parameter over the time increment and obtain the revised values for these for the end of the time increment. The remaining depth-storage curve parameter values could be estimated in a manner similar to the initial estimate of the depth-storage curve parameters by using the equations presented by Mitchell and Jones (1976).

The methods discussed previously could be used in successive time increment iterations to provide a continuing estimate of the depth-storage relationship of the micro-relief surface depression storage.

\section{Example Application}

A simple computer model was written to calculate surface storage, infiltration, and rainfall excess during the course of a recorded rainfall event. The model computed each element at time increments defined by a change in rainfall intensity. Infiltration capacity was used to calibrate the model to runoff records and maximum soil surface depth was varied for comparison. The results obtained from this model were infiltration rate and cumulative volume. cumulative volume of rainfall excess, and the surface storage used at each time increment during the storm.

Three storms, representing a range in duration from 10 to 56 hours and in precipitation from 2.58 to 5.77 inches and having one or more high intensity-short duration periods, were selected from the Allerton watersheds in Piatt County, Illinois. The rainfall events were selected from spring rainfall records because the surface depression storage functions were developed from bare soil data and Central Illinois watersheds are bare in spring.

The infiltration function was defined using the Horton equation (Horton, 1939):

$$
f=f_{c}+\left(f_{o} \cdots f_{c}\right) e^{-k t}
$$

where:

$\begin{array}{lll}\mathrm{f}= & \text { infiltration rate, inches per hour, } \\ \mathrm{f}_{\mathrm{c}}= & \text { innal infiltration rate, inches per hour, } \\ \mathrm{f}_{\mathrm{O}}= & \text { initial infiltration rate, inches per hour, } \\ \mathrm{e}= & \text { base of natural logarithms, } \\ \mathrm{k}= & \text { a constant, and } \\ \mathrm{t}= & \text { time, hours. }\end{array}$

Lytle (1955) determined values for the parameters of the Horton equation for the Allerton watersheds which have soils of the Drummer-Flanagan series. His data were used to obtain estimates of the initial infiltration rate, $\mathrm{f}_{\mathrm{o}}$, and the constant, $\mathrm{k}$, for an equation of:

$$
f=f_{c}+16 e^{-4.68 t}
$$


Equation (105) was used in the model, varying $f_{c}$ to calibrate the model to fit the recorded runoff.

The equations used by Mitchell and Jones (1976) to determine the depth-storage curve parameters $\underline{a}$ and $\underline{b}$ are as follows:

$$
\begin{aligned}
\log a=-0.65840 & -0.12877 D_{m} \\
\log b=0.46046 & -0.38039 a+0.18421 \log a \\
& -0.33797 \log \left(a_{m}\right)
\end{aligned}
$$

where:

$\mathrm{a}$ and $\mathrm{b}$ are the parameters for Equation (2), and

$\mathrm{D}_{\mathrm{m}}=$ maximum micro-relief depth or height, inches.

Equation (2) was used to determine the maximum storage value, $S_{m}$. using the maximum depth value, $D_{m}$. The depth at which runoff would begin, $C$, was assumed to be the equal cut-and-fill depth (Mitchell and Jones, 1976) and was determined by the equation:

$$
\mathrm{C}=\mathrm{D}_{\mathrm{m}}-\mathrm{S}_{\mathrm{m}}
$$

Because the Allerton watersheds have soils of the Drummer-Flanagan soil series, the change in maximum depth during a time increment was determined using Equation (7) from Table 6 . The value of the change in maximum depth, $\Delta \mathrm{D} / \mathrm{D}_{\mathrm{O}}$. multiplied by the maximum depth at the start of the time increment was added algebraically to the initial maximum depth to obtain the maximum depth at the end of the time increment. Equations (106) and (107) were then used to obtain the depth-storage curve parameters a and $\underline{b}$ at the end of the time increment or at the start of the next time increment.

When infiltration rate exceeded rainfall intensity, no storage accumulated. When rainfall intensity was less than infiltration capacity and surface storage had previously accumulated, infiltration capacity was assumed over one-half the surface and the rainfall intensity was the infiltration rate on the remainder. When surface storage volume was exceeded, the volume exceeded was recorded as rainfall excess. No attempt was made to route this excess rainfall to a watershed outlet by overland flow.

For calibration purposes, the model was first run with an initial maximum depth of six inches and the final infiltration rate. $f_{c}$, of Equation (105) was varied to obtain a rainfall excess volume approximately equal to the recorded runoff volume from one of the Allerton watersheds. Then the initial maximum depth, $\mathrm{D}_{\mathrm{m}}$, was varied from 0.0 to 12.0 inches to provide a comparison of the effects of different initial maximum depths. In all of the runs, the time increments correspond to the periods of rainfall intensity, with a change in rainfall intensity dictating the beginning and end of a time increment.

The results of several runs of the model for the three storms are presented in Table 11 . The initial maximum depth, $\mathrm{D}_{\mathrm{m}}$, lists the starting conditions for the micro-relief depth, and the final $D_{m}$ is the maximum micro-relief depth at the end of the storm. The depth above which rainfall excess was accumulated, $\mathrm{C}$, is listed for initial and final conditions 
TABLE: 11. Effect of Initial Maximum Depth and Infiltration Rate on Watershed Model.

\begin{tabular}{|c|c|c|c|c|c|c|c|c|c|c|c|}
\hline $\begin{array}{c}\text { Initial } \\
\mathrm{D}_{\mathrm{m}}^{*} \\
\text { (inches) }\end{array}$ & $\begin{array}{c}\text { Initial } \\
C^{*} \\
\text { (inches) }\end{array}$ & $\begin{array}{c}\text { Final } \\
\mathrm{D}_{\mathrm{m}} * \\
\text { (inches) }\end{array}$ & $\begin{array}{c}\text { Final } \\
C^{*} \\
\text { (inches) }\end{array}$ & $\begin{array}{c}f_{c}^{*} \\
(\mathrm{iph})\end{array}$ & $\begin{array}{c}\text { Max. S* } \\
\text { Used } \\
\text { (inches) }\end{array}$ & $\begin{array}{l}\text { Volume } \\
\text { Infil. } \\
\text { (inches) }\end{array}$ & $\begin{array}{l}\text { Volume } \\
\text { Rainfall } \\
\text { Excess } \\
\text { (inches) }\end{array}$ & $\begin{array}{c}f_{c}{ }^{*} \\
(i p h)\end{array}$ & $\begin{array}{c}\text { Max. S* } \\
\text { Used } \\
\text { (inches) }\end{array}$ & $\begin{array}{l}\text { Volume } \\
\text { Infil. } \\
\text { (inches) }\end{array}$ & $\begin{array}{c}\text { Volume } \\
\text { Rainfall } \\
\text { Excess } \\
\text { (inches) }\end{array}$ \\
\hline
\end{tabular}

STORM 1

\begin{tabular}{|c|c|c|c|c|c|c|c|c|c|c|}
\hline 0.00 & 0.00 & 0.00 & 0.00 & 0.06 & 0.00 & 1.14 & 1.44 & 0.00 & 1.66 & 0.92 \\
\hline 2.00 & 0.17 & 0.30 & 0.00 & & 0.10 & 1.30 & 1.28 & 0.10 & 1.78 & 0.80 \\
\hline 4.00 & 0.22 & 0.59 & 0.02 & & 0.17 & 1.38 & 1.20 & 0.17 & 1.89 & 0.70 \\
\hline 5.00 & 0.29 & 0.74 & 0.04 & & 0.18 & 1.42 & $1.16^{* *}$ & 0.18 & 1.92 & 0.66 \\
\hline 6.00 & 0.39 & 0.89 & 0.07 & & (). 19 & 1.45 & 1.13 & (). 19 & 1.94 & $\left(0.64^{*}\right.$ \\
\hline 7.00 & 0.51 & 1.03 & 0.10 & & 0.20 & 1.48 & 1.10 & 0.20 & 1.95 & 0.63 \\
\hline 8.00 & 0.64 & 1.18 & 0.12 & & 0.22 & 1.50 & 1.08 & (). 22 & 1.96 & 0.62 \\
\hline 10.00 & 0.90 & 1.48 & 0.15 & & 0.26 & 1.53 & 1.115 & 0.25 & 1.97 & 0.61 \\
\hline 12.00 & 1.12 & 1.77 & 0.17 & & 0.30 & 1.54 & $1 .(14$ & 0.30 & 1.98 & 0.60 \\
\hline
\end{tabular}

\begin{tabular}{|c|c|c|}
\hline Vo & 0.00 & 0.00 \\
\hline & 2.00 & 0.17 \\
\hline & 4.00 & 0.22 \\
\hline & 5.00 & 0.29 \\
\hline & 6.00 & 0.39 \\
\hline & 7.00 & 0.51 \\
\hline & 8.00 & 0.64 \\
\hline & 10.00 & 0.90 \\
\hline & 12.00 & 1.12 \\
\hline
\end{tabular}

Date: $5-27-55$, Duration $=10.42$ hours, Precipitation $=2.22$ inches, Maximum Intensity $=5.00$ iph

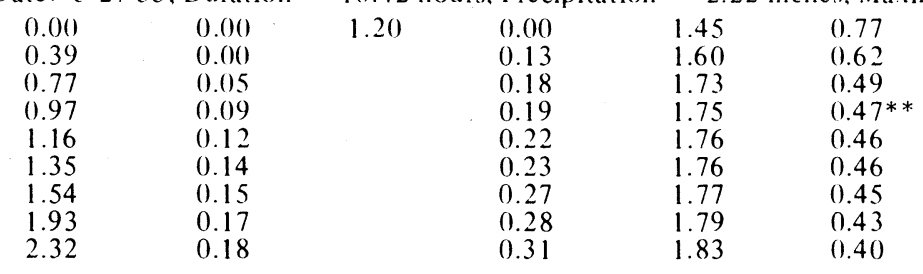

STORM 3

Date: $4-19-64$, Duration $=56.02$ hours, Precipitation $=5.77$ inches, Maximum Intensity $=5.40 \mathrm{iph}$

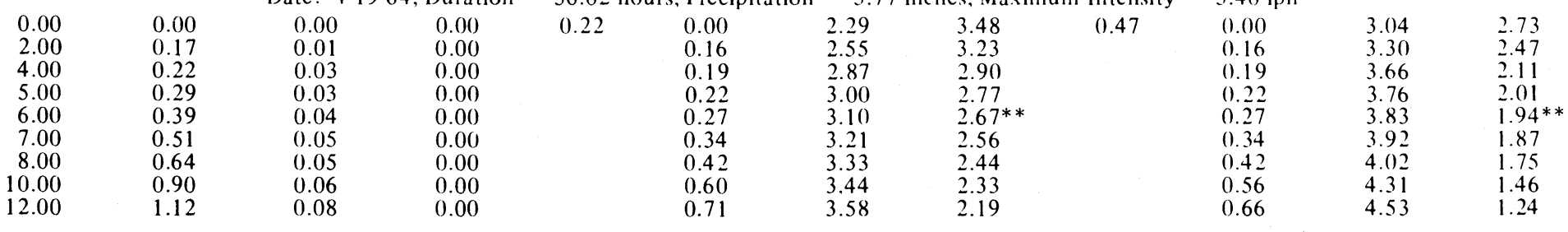

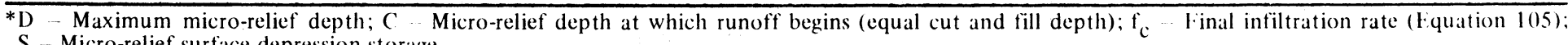
$\mathrm{S}$ - Micro-relief surface depression storage.

** Model run at which the volume of rainfall excess equals the runoff volume measured from one of two Allerton watersheds. 
to aid in visualizing the portion of maximum depth actually available for storage before runoff could begin.

Two conditions for the final infiltration rate. $f_{c}$. of Equation (105) are presented for two of the storms. As described previously. $f_{c}$, for an initial $D_{m}$ of six inches. was varied until the cumulative rainfall excess approximately equaled the recorded runoff volume from one of the Allerton watersheds. The $f_{c}$ conditions for two of the storms are the $f_{c}$ values needed to calibrate the model to the runoff from each of the two Allerton watersheds. Storm 2 has only one $f_{c}$ condition because runoff records are complete for only one watershed for that storm.

Table 11 lists the maximum micro-relief storage used. the cumulative volume of infiltration, and the cumulative volume of rainfall excess. The maximum $S$ used is not necessarily the maximum storage available during the storm: in fact the maximum storage available near the first of a storm was not used. The double asterisks in Table 11 designate those runs where the volume of rainfall excess from the model equals the volume of runoff measured from one of the two Allerton watersheds.

The effect of depression storage. as compared with no depression storage. on the volume of runoff and infiltration is affected by storm duration. The short duration storm (Storm 2) demonstrates much less reduction in runoff (increase in infiltration) than the longer duration Storms 1 and 3. A series of model calculations with the last 13-hour portion of Storm 1 confirmed this phenomenon when compared with Storms 1 and 3. As would be expected. infiltration rate has a marked effect on runoff. However, the runoff amount is reduced with increased initial maximum storage depth. The relative reduction in runoff observed by comparing between storms and between final infiltration rates is difficult to discern. Surface storage is part of a dynamic process which is dependent upon infiltration rate and storm pattern as well as many other factors.

The details of one $f_{c}$ condition for each of the three storms of Table 11 were plotted for visual comparison. Figures 2 through 4 show the computed cumulative infiltration. cumulative volume of rainfall excess, the surface storage used during the storm. and the measured cumulative runoff from one of the watersheds. The computed cumulative rain fall excess and watershed runoff curves are of interest because of their similarities. For the storm of April 22 and 23, 1952 (Figure 2), the rainfall excess and watershed runoff curves begin at the same time. In Figures 3 and 4 the rainfall excess and watershed runoff curves have a starting discrepancy of only 0.21 and 0.33 hours, respectively. No time-scale shifting was made between the model simulation and the watershed runoff data. The general configuration of the excess and watershed runoff curves is similar if one visualizes an overland flow delay between the two curves.

Figures 2 through 4 also illustrate that micro-relief surface depression storage is available for several abstractions from runoff with the kind of rainstorms experienced in central Illinois. Even in the case of the storm of May 27. 1955. which had two rather intense early bursts of rainfall (Figure 3), micro-relief storage was available for a second abstraction, thereby retarding runoff. This is in contrast to many runoff determination methods, which assume only an initial abstraction by the micro-relief surface depression.

This rainfall-runoff model calculation demonstrates the value of including the microrelief surface depression storage as a dynamic function in a watershed model. The changes in micro-relief storage may be included in a model with a minimum of effort, as demonstrated in this example. 


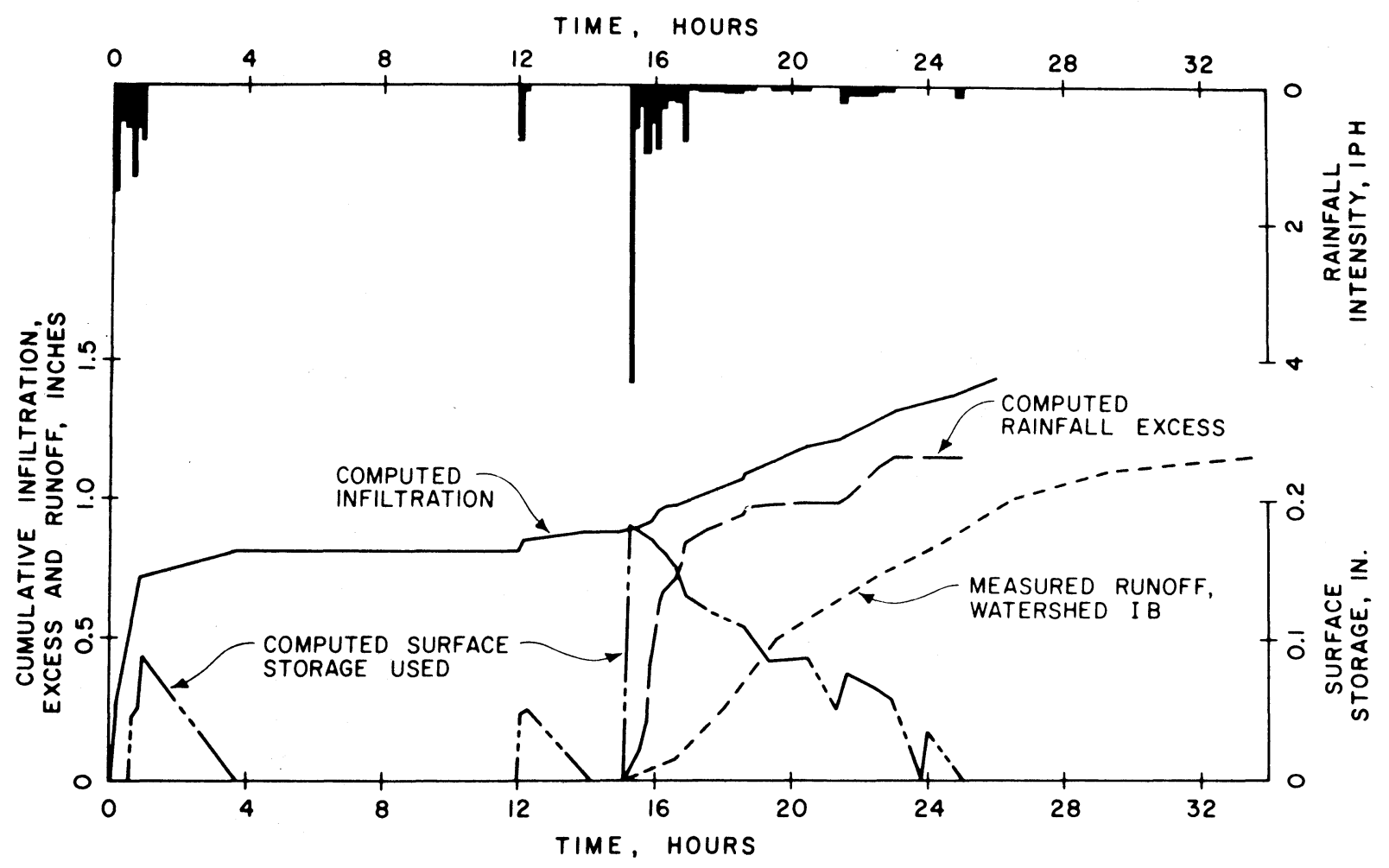

Figure 2. Result of Modeling Storm of April 22 and 23, 1952, and Measured Runoff from Allerton Watershed 1B. (Initial $\mathrm{D}_{\mathrm{m}}=5.0$ inches, $\mathrm{f}_{\mathrm{c}}=0.06 \mathrm{iph}$ ) 


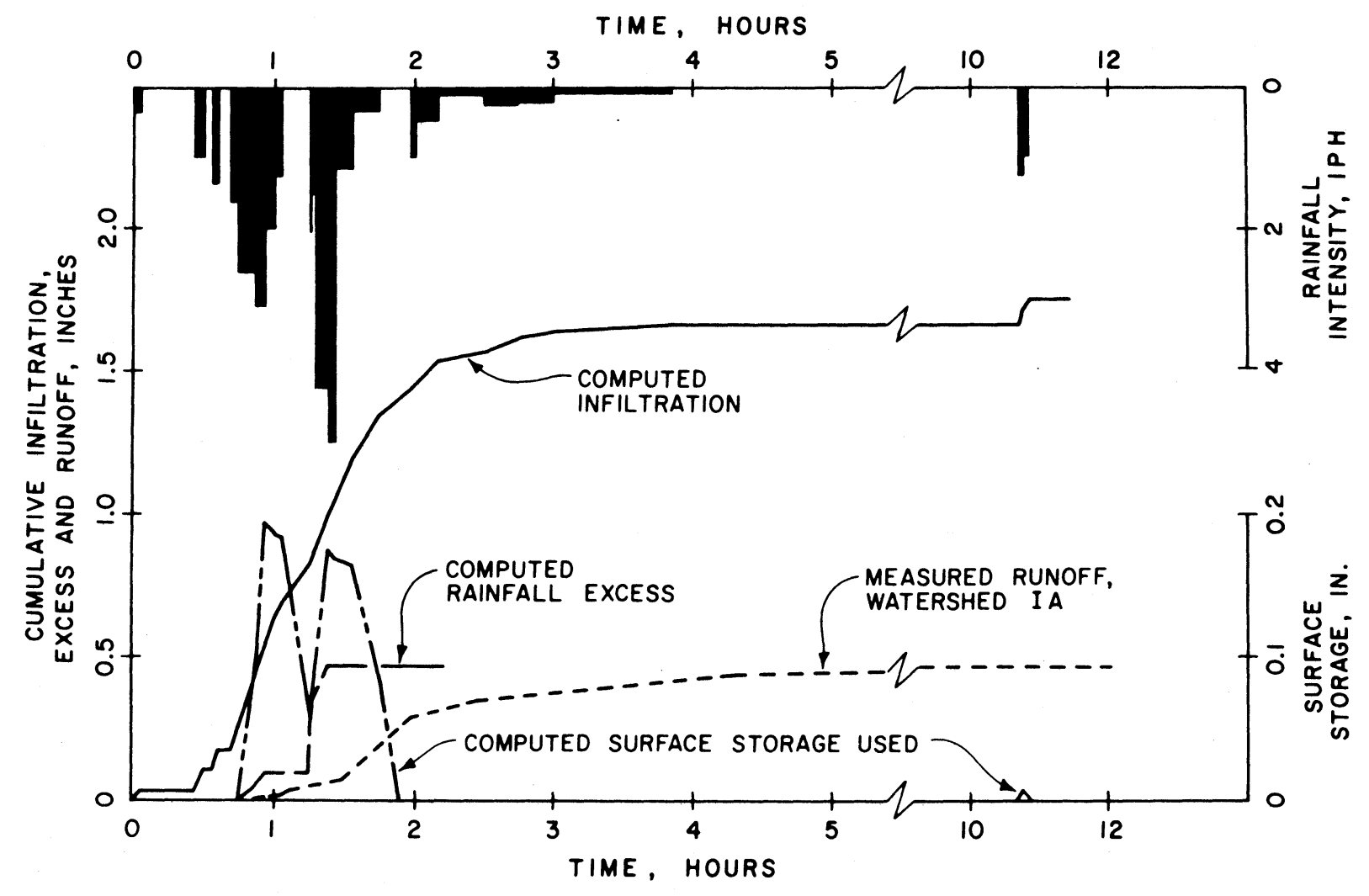

Figure 3. Result of Modeling Storm of May 27, 1955, and Measured Runoff from Allerton Watershed 1A. (Initial $\mathrm{D}_{\mathrm{m}}=5.0$ inches, $\mathrm{f}_{\mathrm{c}}=120 \mathrm{iph}$ ) 


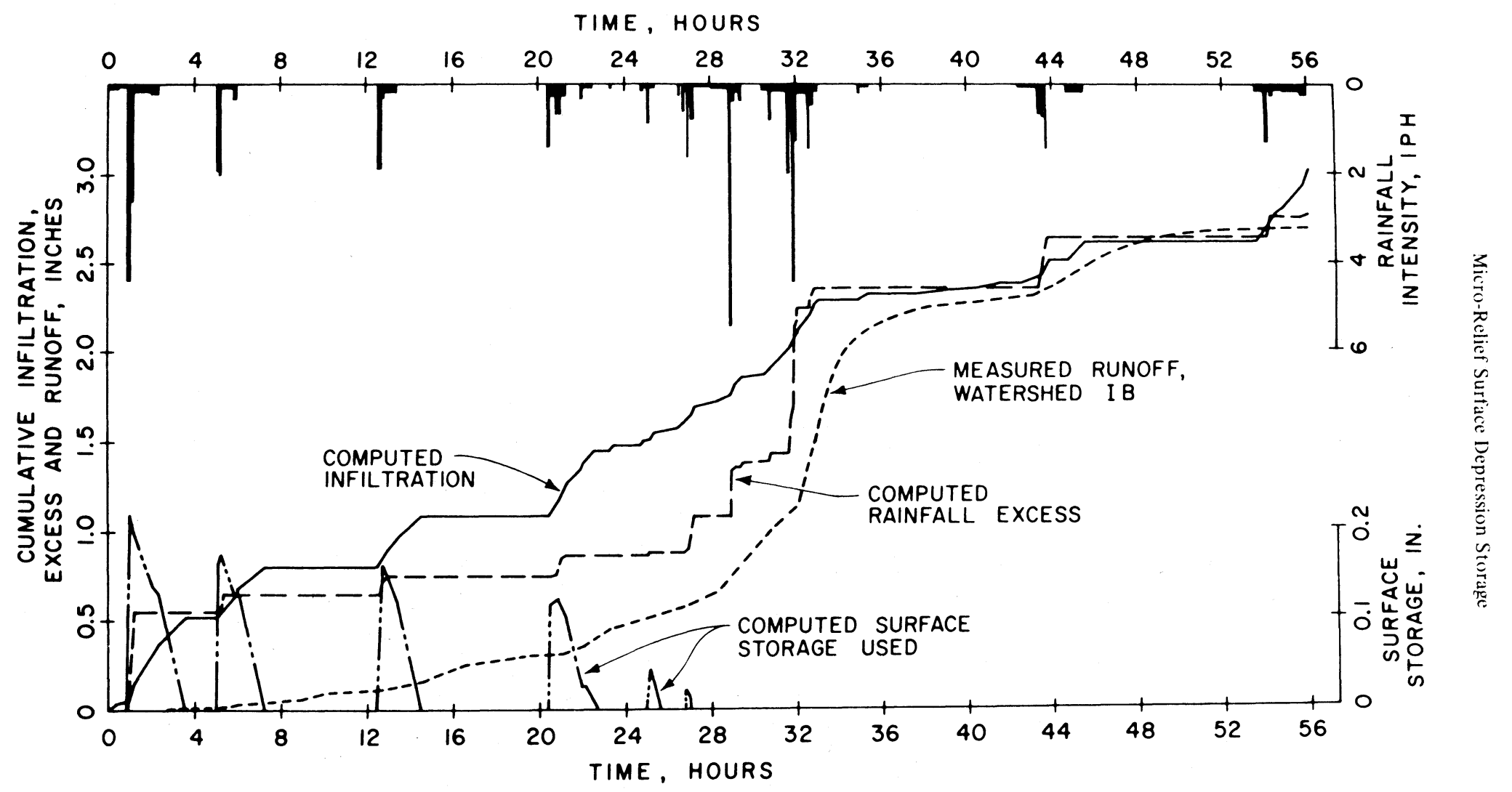

Figure 4. Result of Modeling Storm of April 19-21, 1964, and Measured Runoff from Allerton Watershed 1B.

(Initial $\mathrm{D}_{\mathrm{m}}=6.0$ inches, $\mathrm{f}_{\mathrm{c}}=0.22 \mathrm{iph}$ ) 


\section{SUMMARY}

The objectives of the study were to quantify the change in micro-relief surface depression storage during a rainfall event; to relate the changes to rainfall, hydrologic, and soil parameters; and to demonstrate how the change in micro-relief storage functions can be applied. Micro-relief measurements were made on 88 soil bin samples from four soils before and after the application of simulated rainfall. The rainfall varied from 3 to 15 inches per hour in intensity and from 10 to 50 minutes in duration. The data were analyzed separately by soil types and as a group.

Empirical relationships were developed to describe the change in micro-relief surface depression storage during a rainfall event. The surface depression storage changes were adequately described with linear equations that used basic rainfall, surface hydrology, and soil parameters and cross products of these parameters as independent variables. The dependent variables in these equations were the changes in the maximum micro-relief depth, in the micro-relief depression storage, and in the depth-storage model parameters a and $\underline{b}$.

The prediction equations may be used in a watershed model that iterates in successive time increments within discrete subareas of the watershed. A rainfall-runoff model developed for demonstration purposes was used with three storms ranging from 2.22 to 5.77 inches of precipitation and from 10.42 to 56.02 hours duration. The results of this simulation demonstrated agreement between simulated rainfall excess and measured watershed runoff and illustrated the manner in which micro-relief surface depression storage functions during a rainfall event.

\section{LITERATURE CITED}

Bubenzer, G. D., 1970. Effect of Drop Size and Impact Velocity on the Detachment of Soils Under Simulated Rainfall. Unpublished Ph.D. Thesis. Library, University of Illinois at UrbanaChampaign.

Bubenzer, G. D. and B. A. Jones, Jr., 1971. Drop Size and Impact Velocity Effects on the Detachment of Soils Under Simulated Rainfall. Trans. ASAE 14:625-628.

Crawford, N. H. and R. K. Linsley, 1966. Digital Simulation in Hydrology: Stanford Watershed Model IV. Dept. of Civil Engineering Tech. Report No. 39, Stanford University, Stanford, California.

Holtan, H. N., G. J. Stiltner, W. H. Henson, and N. C. Lopez, 1975. USDAHL-74. Revised Model of Watershed Hydrology. USDA-ARS Tech. Bulletin No. 1518.

Horton, R. E., 1939. Analyses of Runoff-Plot Experiments with Varying Infiltration-Capacity. Trans. Amer. Geo. Un. 20:693-711.

Huggins, L. F. and E. J. Monke, 1966. The Mathematical Simulation of the Hydrology of Small Watersheds. Purdue University Water Resources Center Tech. Report No. 1, Lafayette, Indiana.

Lytle, W. F., 1955. Determination of infiltration values from small watersheds. Unpublished Report. In: Files of Research Project 312 - "Runoff from Small Agricultural Watersheds in Illinois." Department of Agricultural Engineering, University of Illinois at Urbana-Champaign.

Massie, L. R., 1975. An Upland Erosion Model. Unpublished Ph.D. Thesis. Library, University of Wisconsin-Madison.

Mitchell, J. K., 1970. Micro-Relief Surface Depression Storage. Unpublished Ph.D. Thesis. Library, University of Illinois at Urbana-Champaign.

Mitchell, J. K. and B. A. Jones, Jr., 1973. Profile Measuring Device. Trans. ASAE 16:546-547.

Mitchell, J. K. and B. A. Jones, Jr., 1976. Micro-Relief Surface Depression Storage: Analysis of Models to Describe the Depth-Storage Function. Water Resources Bulletin 12:1205-1222. 


\section{AMERICAN WATER RESOURCES ASSOCIATION}

St. Anthony Falls Hydraulic Laboratory

Mississippi River at Third Avenue S.E.

Minneapolis, Minnesota 55414

(612) $376-5050$

\section{MEMBERSHIP INFORMATION}

The American Water Resources Association (AWRA) is a scientific and educational non-profit organization established to encourage and foster interdisciplinary communication among professionals of diverse backgrounds working on all aspects of water resource problems.

Professional persons, agencies, and corporations interested in any aspect of water resources can benefit greatly from membership in AWRA.

The principal objectives of AWRA are:

- the advancement of water resources research, planning, development, management, and education.

- the establishment of a common meeting ground for physical, biological, and social scientists, engineers, and other persons concerned with water resources.

- the collection, organization, and dissemination of ideas and information in the field of water resources science and technology.

For further information write to the American Water Resources Association at its headquarters in Minneapolis.

\section{WATER RESOURCES BULLETIN \\ (Bimonthly - starting with February)}

This journal is primarily dedicated to the publication of original and review papers, characterized by their broad approach to water resources problems, solutions, and policy. All papers are reviewed by fellow scientists prior to publication. Each volume contains approximately 1200 pages and includes 125 articles, book reviews, water news, and meeting announcements. Editor: William R. Boggess. 
October 24, 1978

Errata

Mitchell, J. K. and Jones, B.A., Jr. 1978. Micro-relief surface depression storage: changes during rainfall events and their application to rainfall-runoff models. Water Resources Bulletin 14: 777 - 802 .

Page 797, Table 11. The first four column heads should be:

Initial

$\mathrm{D}_{\mathrm{m}}{ }^{*}$

(inches)
Initial

$S_{C} *$

(inches)
Final

$\mathrm{D}_{\mathrm{m}}$ *

(inches)
Final

$S_{C}$ *

(inches).

The footnote should be:

* D-Maximum micro - relief depth; $S_{C}$ - Micro - relief storage at the depth at which runoff begins (equal cut and fill depth); $f_{c}-$ Final infiltration rate (Equation 105); $s$ - Micro - relief surface depression storage. 